\title{
HEMATOMAS RENALES POSTLITOTRICIA EXTRACORPÓREA EN 324 SESIONES CONSECUTIVAS CON EL LITOTRITOR DOLI - S: INCIDENCIA, CARACTERÍSTICAS, ANÁLISIS MULTIFACTORIAL Y REVISIÓN
}

\author{
Rodolfo Orozco Fariñas, José Ignacio Iglesias Prieto, Jorge Massarrah Halabi, José Ma Mancebo \\ Gómez y Enrique Perez-Castro Ellendt.
}

Unidad de Urología. Clínica La Luz. Madrid. España.

\begin{abstract}
Resumen.- OBJETIVO: Determinar la prevalencia de los hematomas renales postlitotricia en nuestra unidad, la incidencia de los sintomáticos y/o progresivos, el comportamiento clínico, el manejo de los mismos, asi como los factores potencialmente influyentes sobre dichos aspectos.
\end{abstract}

MÉTODO: Estudio observacional prospectivo en el que analizamos diversos parámetros integrados en nuestra base de datos a partir de hojas recopilatorias confec- cionadas en 314 pacientes sometidos a litotricia sobre 324 unidades renales en igual número de sesiones. Para el análisis estadístico se utilizó el paquete SPSS 15.0 con la asesoría de especialistas en bioestadística. Para el diagnóstico de los hematomas se utilizaron datos clínicos (interrogatorio y exploración física), hematológicos y ecográficos, estos últimos en casos aislados complementados con TAC, se realizó seguimiento mediante contacto telefónico con los pacientes durante un periodo de tiempo comprendido entre los 7 y los 19 meses después de la litotricia.

RESULTADOS: La prevalencia de hematomas fue del $13 \%$ pero sólo el 6,2 \% fueron sintomáticos, la incidencia acumulada de hematomas de evolución progresiva fue de el 2,16\% y en el 0,92 \% de las litotricias se requirió transfusión sanguínea a causa de los hematomas, lo que representó el 7,14\% de estos.

Los factores estadísticamente asociados a la incidencia de hematomas fueron el número de onda (superior a 2300), la energía total (a partir de $150 \mathrm{Jl}$, el KV la partir de 17,5), la microhematuria preoperatoria, la hipertensión perioperatoria, la litiasis de cistina, el hidrocáliz, la localización calicial del cálculo, Iprincipalmente inferior), la asociación de cardiopatía isquémica con hipertensión arterial, (HTA) o hepatopatías, las hepatopatías crónicas perse, la elevación de transaminasas, el uso habitual de antiagregantes plaquetarios y AINEs sobre todo con pauta previa de heparina fraccionada para la progresión de los hematomas) y la combinación de estos con HTA pre-operatoria lpara los hematomas síntomáticos), así como la presencia de litiasis múltiple tratadas en la misma sesión, encontrándose grados de asociación diferentes para los distintos subgrupos de hematomas establecidos. 
Se observó diferencias en el comportamiento clínico de los tipos de hematomas (subcapsular y perirrenal) y el $12 \%$ de los pacientes con hematomas permanecieron sintomáticos entre 2 y 6 meses después.

CONCLUSIONES: El hematoma renal postlitotricia es mas frecuente de lo que creemos; su magnitud y evolución variables dependen de múltiples factores, algunos de los cuales tienen mayor peso específico (hepatopatías, comportamiento de la tensión arterial perioperatoria y uso habitual de fármacos antiagregantes con necesidad de heparina fraccionada perioperatorial, lo que dió lugar a pacientes con mayor riesgo de presentar hematomas cuando concurrieron 3 o mas de los factores relacionados encontrados en la presente serie. Los hematomas subcapsulares y los perirrenales difieren en su comportamiento clínico. Los hematomas con diámetros ecográficos iniciales igual o mayores de $4 \mathrm{~cm}$ fueron mas propensos a la progresión.

El riesgo de expresión sintomática o de progresión de un hematoma se evidenció en los primeros cinco días postlitotricia aún cuando permanecieran asintomáticos o estables clínica e imagenológicamente en las primeras 24 hrs., todo lo cual aconseja un seguimiento cuidadoso y reposo físico relativo durante la primera semana en los pacientes con mayor riesgo, y considerar el reposo al menos cinco días en todos los pacientes tratados, entre otras medidas.

El dolor lumbar puede persistir hasta seis meses en algunos pacientes después de padecer un hematoma postlitotricia clínicamente significativo.

Palabras clave: Hematoma renal. Litotricia por ondas de choque. Hematomas perirrenales. Hematoma renal subcapsular.

Summary.- OBJECTIVES: The objective is to know the prevalence of renal hematoma after lithotripsy in our unit, as well as the incidence of symptomatic and/or progressive hematomas, their clinical behavior and management, and also the factors potentially influencing those features.

METHODS: A prospective study in wich we analyzed various parameters from the database on 314 patients undergoing SWL on 324 renal units. SPSS 15.01 was employed for statistical analysis under supervision of biostatistics experts. The diagnosis of hematoma was obtained with clinical data (history and physical examination), blood analysis, and ultrasound, this latter with the complement of CT scan in isolated cases. All patients underwent follow-up by means of phone contacts over a period between 7-19 months after lithotripsy.
RESULTS: The prevalence of hematoma was $13 \%$ but only $6.2 \%$ were symptomatic. Accumulated incidence of hematoma with progressive evolution was $2.16 \%$, and blood transfusion requirement due to hematoma was $0.92 \%$ of all lithotripsies, which represents $7.14 \%$ or them.

Factors statistically associated with the incidence of hematoma were: number of shock waves (over 2300), total energy (above 150J), number of KV labove 17.5), preoperative microhematuria, perioperative hypertension, cystine lithiasis, hydrocalyx, caliceal localizations (mainly lower calyces), the association of coronary artery disease with hypertension, or hepatic diseases, chronic hepatopathy, elevation of transaminases, usual intake of anti platelet aggregation drugs and nonsteroidal anti-inflammatory drugs /mainly with prior lowweight heparin treatment for hematoma progression), and a combination of the previous with preoperative hypertension (for symptomatic hematoma), as well as the presence of multiple stones treated in the same session, with different degrees of association for the various subgroups of hematomas.

We observed differences in clinical behavior depending on the type of hematoma Isubcapsular and perirenall and $12 \%$ of the patients with hematoma remained symptomatic between 2 and six months later.

CONCLUSIONS: Renal hematoma after shock wave lithotripsy is more frequent than what is believed. Hematoma's size and evolution depend on multiple factors some of them with a greater weight (hepatopathy, perioperative blood pressure behavior, usual intake of anti-aggregation drugs with need of perioperative low weight heparin) which a greater risk when three or more factors concurred. Subcapsular and perirenal hematomas have a different clinical behavior. Initial ultrasound hematoma diameters equal $\geq 4 \mathrm{~cm}$ were more prone to progression.

The risk of symptomatic clinical presentation or progression was evident within the first five days after shockwave lithotripsy even when asymptomatic or clinically/radiologically stable over the first 24 hours, therefore, careful follow-up and physical resting is counselled over the first week in patients with high risk, and relative physical resting during at least five days in all patients undergoing treatment. Lumbar pain may persist up to six months in some patients with renal clinically significant hematoma after shockwave lithotripsy.

Keywords: Renal hematoma. Shockwave lithotripsy. Perirenal hematoma. Subcapsular renal hematoma. 


\section{INTRODUCCIÓN}

Hace 27 años irrumpió en el arsenal terapéutico urológico, con el primer paciente tratado por el Dr. Chaussy, la litotricia extracorpórea por ondas de choque, un hito que fue producto del avance tecnológico y la capacidad científica del hombre al servicio la medicina; la universalización de dicho tratamiento y consecuentemente, su primera aplicación en España por los Dres. Ruiz Marcellan e lbarz Servio, se produjo en el año 1984 (1), y su lugar hegemónico en el ámbito terapéutico de la enfermedad litiásica urinaria es actualmente incuestionable, haciendo que, con el soporte de técnicas como la ureterorrenoscópia, la nefrolitotomía percutánea, o la simple colocación de un catéter "doble J", sea muy infrecuente, en la actualidad, la necesidad de que pacientes urolitiásicos precisen técnicas mas invasivas para su solución.

El reconocimiento y afianzamiento de esta técnica se debe a su perfeccionamiento constante en función de mayor sencillez, comodidad, precisión, flexibilidad, eficacia, calidad de imágenes radiológicas y/o ultrasonográficas durante el proceder, $y$, sobre todo, mínima morbilidad.

Nuestro trabajo va dirigido a este último aspecto y específicamente hacia una de las complicaciones mas importantes; es el caso de los hematomas renales postlitotricia. Si bien es cierto que en orden de frecuencia esta complicación ocupa en las grandes series entre el tercer y el cuarto puesto, después del cólico nefrítico, la uropatía obstructiva severa y la urosepsis (1), no podemos olvidar que, junto a esta última, y otras de carácter excepcional, conforma un grupo de complicaciones potencialmente mortales, sobre todo en pacientes de alto riesgo (2-6).

De acuerdo con las investigaciones realizadas, el hematoma renal postlitotricia se produce por pequeñas laceraciones o daño parietal sobre la microvasculatura renal (7-9), cuya morfología serpenteante la hace vulnerable (10), al parecer las vénulas arciformes son las mas propensas a estas lesiones, pero las arteriolas también pueden lesionarse, estas tendrían como consecuencia hematomas renales de mayor gravedad según hemos podido comprobar mediante arteriografía, (caso comentado mas adelante en este apartado), también en hematomas perirrenales se ha descrito lesión de arterias capsulares (11). La rotura de la cápsula en un hematoma originalmente subcapsular ha sido observada en la presente serie.

Después de revisar la literatura médica específica puede comprobarse que la incidencia de los he- matomas renales reportada por los autores realmente depende de los criterios y técnicas de diagnóstico, la relevancia semiológica de dichos hematomas, y el tipo de estudio científico realizado (experimental, observacional, retrospectivo o prospectivo, etc.), lo que nos lleva a pensar que se trata de una complicación cuya incidencia puede conllevar sesgos estadísticos que pueden distorsionar la apreciación que tenemos de la misma, situación que entraña el peligro, siempre indeseable en la práctica médica, de la infravaloración.

La Unidad de Litotricia de la Clínica "La Luz", en la cual se ha llevado a cabo el presente estudio, fue fundada en julio de 1985 por el Dr. Enrique Perez-Castro Ellendt quien la dirige actualmente, desde entonces se han realizado 15.000 litotricias renoureterales extracorpóreas por ondas de choque con diferentes tipos de litotritores que se han sucedido conforme al desarrollo técnico y científico de los mismos, así pués, los profesionales que han trabajado en ella a lo largo de estos veintidós años han utilizado las máquinas HM3, HM3 modificada, MPL 9000, MFL 5000 y el actual DOLI-S en los últimos tres años. Con todos ellos se ha acumulado una experiencia que nos permite enfrentar las diversas exigencias que dicha labor asistencial nos demanda en la práctica diaria; no obstante, paralelamente, siempre se ha considerado ineludible en nuestra unidad la labor científico investigativa ante los problemas que se han ido presentando para llegar a conclusiones mejor sustentadas que permitieran al menos minimizar sus consecuencias, es por ello que a raíz de la aparición en uno de nuestros pacientes de un hematoma renal grave, de evolución progresiva, refractario a las medidas conservadoras, solucionado finalmente mediante embolización selectiva de una arteriola interlobar, nos propusimos realizar un estudio prospectivo observacional descriptivo para determinar la frecuencia de esta complicación, la importancia clínica en virtud de su comportamiento evolutivo, su manejo terapéutico y los posibles factores condicionantes o predisponentes.

El análisis de estos aspectos es el contenido principal de este trabajo; así mismo, hemos realizado una amplia revisión de la literatura médica especializada desde el advenimiento de la litotricia extracorpórea a fin de analizar nuestros hallazgos con la mayor objetividad posible aunque con la relatividad que implican para la comparación, las diferencias entre diseños investigativos, condiciones tecnicoinstrumentales, e incluso regionales (por su influencia sobre la composición litiásica del clima, los hábitos alimenticios, las características del agua bebible y las condiciones socioeconómicas). 


\section{MATERIAL Y MÉTODO}

El equipo litotritor utilizado fué el litotritor Dornier S (DOLI-S), fabricado en el año 2004, con generador electromagnético del tipo 220 f XXP con las siguientes caracteristicas técnicas:

- Angulo de apertura: $73^{\circ}$

- Pr focal: de 49 a $90 \mathrm{MPa}$ (megapascales).

- Energía focal (12 mm): de 16 a $110 \mathrm{~mJ}$.

- Densidad de energía: de 0,35 a 1,9 mJ / mm2.

- Foco (diámetros axial/lateral): $27 / 63 \mathrm{~mm} x$ 2,7/5,4 mm.

- Ocho niveles de energía para las ondas de choque hasta los $20 \mathrm{Kv}$ con ajuste automático de las variables anteriores según el nivel empleado.

De acuerdo con las características resumidas con anterioridad los valores de las variables terapéuticas utilizadas en nuestros pacientes fueron: número de impactos de ondas aplicado 2767,39 +/- 865,03 (mín. 1010, max. 4660), intensidad de onda $17,684+/-0,524$ (16,3-19 KV o niveles entre 8 y 13 de display), energía total 190,39+/- 0,98
$(55-405 \mathrm{~J})$, frecuencia entre 60 y 80 ondas/min, energía focal de 68 a $93 \mathrm{~mJ}$, densidad de energía 1,3 a 1,7 mJ / mm2 y foco de $60 \times 4,1 \mathrm{~mm}$. En mas del $90 \%$ de los pacientes se aplicó una frecuencia de impactos de $80 / \mathrm{min}$. por lo que los datos tecnicoterapéuticos verdaderamente factibles, (ver discusión), para el análisis estadístico fueron fueron: intensidad, número de ondas y energía total.

El universo de estudio fué el de los pacientes tratados por litiasis renal de cualquier localización y/o de uréter proximal a nivel de L2-L3, con independencia del número y repercusión anatomofuncional $y$, obviamente, sin contraindicaciones absolutas para el proceder en sí, (Cuadro I); no se excluyeron casos según edad, sexo, raza y características antropométricas.

La muestra quedó conformada por 324 unidades renales expuestas a LEOCH en 314 enfermos entre 1 y 84 años de edad $(51,2+/-15$ años), de los cuales la distribución por sexo fue de $161(51,2$ $\%)$ mujeres y $153(48,7 \%)$ hombres. La distribución por unidad renal expuesta fue de $144(44,4 \%$.) en

\section{CUADRO I. CONTRAINDICACIONES DE LA LITOTRICIA EXTRACORPÓREA POR ONDAS DE CHOQUE.}

- Anomalías de la coagulación no controladas.

- Tratamiento con antiagregantes plaquetarios vigente.

- Tumores renales. *

- Nefrítis o pielonefrítis aguda, absceso renal.

- Hepatoesplenomegalia. *

- Insuficiencia renal severa.

- Quiste hidatídico renal o hepatoesplénico (según tamaño y localización). **

- Embarazo avanzado.

- Deformidad osteoesquelética (somática o neurogénica) u obesidad mórbida que impida la focalización.

- Obstrucción de vías altas, distales a la litiasis, sin derivación o solución previa.

- Arritmias cardíacas inestables o incontroladas.

- Litiasis coraliforme completa o compleja sin técnicas complementarias (p.ej. NLP, cirugía, cateter

"doble J", etc.).

- Insuficiencia cardiorrespiratoria severa.

- Infección severa respiratoria o de otros sistemas.

* relativas por potenciales hematomas postlitotricia, (¿́prioridad de la litiasis?)

* relativa por potenciales complicaciones. 
lado derecho y $180(55,6 \%)$ en el lado izquierdo. El $71 \%$ de los pacientes eran portadores de litiasis única mientras que el $29 \%$ tenían 2 o más cálculos.

Se confeccionaron impresos de registro de datos para cada paciente y sesión con el fin de recopilar datos clínicos pre y postlitotricia (antecedentes litiásicos, enfermedades asociadas, fármacos concomitantes, presencia de uropatía obstructiva, sintomatología en las 22 a $24 \mathrm{hrs}$. siguientes a la litotricia y comportamiento de la TA pre y postlitotricia), antropométricos (para determinar el índice de masa corporal o IMC), caracterización de la/s litiasis (número, localización, tamaño), detalles tecnicoterapéuticos (intensidad o KV, número de ondas, frecuencia y energía acumulada), datos de laboratorio (hemograma, creatinina, transaminasas, estudio de coagulación, urocultivo y sistemático de orina), y ecográficos.

Todos los datos fueron introducidos en el paquete informático estadístico SPSS. 15.0 para su procesamiento a fin de establecer frecuencias y relaciones entre las variables para el correspondiente análisis global y específico, para dicho fin nos apoyamos en las pruebas de Chi cuadrado, Fisher, ANOVA, correlaciones de Spearman, Test de Student y Regresión logística para la valoración del tipo de relación estadística y la fuerza de la misma teniendo en cuenta la frecuencia de las variables analizadas y sus características (categóricas, no categóricas, binomiales, multinomiales, dependientes, independientes, cofactores, etc.), así como el objetivo a esclarecer; es por ello que en algunos subgrupos se aplicaron test no paramétricos al tratarse de subgrupos de bajas frecuencias.

Los dos grandes grupos comparativos fueron: "pacientes con resultado de hematoma" $(\mathrm{H})$ y "pacientes sin resultado de hematoma"; a su vez, el grupo $\mathrm{H}$ para su mejor análisis fue subdividido en tres categorías, estas fueron, pacientes con hematomas asociados a síntomas ("hematomas sintomáticos o HS"), pacientes asintomáticos ("hematomas asintomáticos o $\mathrm{HA}^{\prime \prime}$ ), y pacientes con hematomas con evolución progresiva de su tamaño ("hematomas progresivos o $\mathrm{HP}^{\prime \prime}$ ); en este último fueron incluídos también los detectados en el seguimiento de los hematomas asintomáticos y aquellos que requirieron atención médica después del alta por síntomas secundarios al incremento del hematoma. Todos los grupos fueron comparados entre sí para las diferentes variables, pero las que carecieron de interés no son expuestas en detalle para aminorar la densidad de datos innecesarios (mas de 20.000 valores analizados); por la misma razón se obvia la tabulación numérica detallada de algunos factores asociados estadísticamente cuyo resultado final se comparó con los reportes de otros autores.

En el subgrupo de HS, se incluyeron aquellos que además del hematoma presentaron algún síntoma que no pudo ser descartado como consecuencia del hematoma aunque tampoco absolutamente atribuible al mismo como las náuseas y los vómitos, no obstante los síntomas determinantes para la inclusión en este grupo fueron: dolor lumbar o de otro tipo no explicable por otras causas, palidez cutáneomucosa, cambios físicos inesperados en la región lumbar, signos de irritación psoica, lipotimia o mareo ortostático, hipotensión arterial, aumento de frecuencia cardíaca y disminución de la $\mathrm{Hb}$ y el hematocrito.

Se realizó un estudio prospectivo, observacional no controlado en base a las 324 sesiones, e igual número de unidades renales expuestas, de litotricia por ondas de choque efectuadas en el período comprendido entre marzo de 2006 y mayo de 2007 en la Unidad de Litotricia de la Clínica La Luz de Madrid. Al tratarse nuestra Unidad de un centro de referencia, cuyo seguimiento al alta corresponde al centro y especialista remisor en la mayoría de los casos, la comprobación evolutiva tras las $24 \mathrm{hrs}$ en estos, solo pudo hacerse por vía telefónica, careciendo de datos objetivos sistemáticos ulteriores en la mayoría de los datos analizados, no obstante, todos los pacientes diagnosticados de hematoma sintomático no fueron dados de alta hasta tenerse la completa seguridad de que tenían una evolución clínica, ecográfica, hematológica y hemodinámica estables, lo cual nos permitió monitorizar y determinar la prevalencia de los hematomas con signos de progresión y/o sintomáticos, en las primeras 22 a 24 hrs. de la litotricia; a posterioris la evolución controlada telefónicamente nos permitió establecer la incidencia acumulada en base a los casos no diagnosticados durante el ingreso por ausencia de signos sugerentes, así como, la evolución de los detectados durante el mismo; es por ello que aunque los resultados para el diagnóstico de hematoma se refieren solo a la prevalencia de estos, para los subgrupos podríamos hablar de incidencia al haberse detectado nuevos casos en el seguimiento realizado tras el alta hospitalaria.

Para el diagnóstico ecográfico del hematoma renal y su medición se siguieron los siguientes criterios:

- Ecogenicidad parenquimatosa disminuída o ecolucidez.

- Interfase ecogénica con parénquima normal.

- Contorno capsular, pericapsular y subcapsular 
- Configuración del seno renal (expandido, comprimido o rechazado).

- Delimitación corticomedular.

- Ecolucidez perirrenal.

- Diámetros (largo y ancho) de hematoma.

- Distancia desde el riñón a la pared muscular (apreciación cualitativa).

- Distancia entre el ren y bazo o hígado (apreciación cualitativa).

- Área elíptica del hematoma $=\pi \times \operatorname{largo} / 2 \times$ ancho/2.

- Eje longitudinal renal.

- Distancia desde interlínea corticomedular a borde renal (parénquima).

Las ecografías fueron realizadas en todos los casos a la mañana siguiente (aproximadamente a las $18 \mathrm{hrs}$. de la litotricia) pero en los pacientes con cuadro clínico sugestivo (dolor lumbar intenso, refractario o atípico asociado o no a signos hipovolémicos) fueron efectuadas en el momento de su aparición realizándose seguimiento aproximadamente cada $8 \mathrm{hrs}$. en los casos cuyo hematoma fuera corroborado ecográficamente o si los síntomas persistían a pesar de ecografía inicial normal, en dicha situación se realizó seguimiento con hemograma cada 8 o $12 \mathrm{hrs}$. En 2 casos se complementó el diagnóstico de hematoma con TAC.

El alta hospitalaria de los pacientes se produjo a las 22 - $24 \mathrm{hrs}$. en todos los asintomáticos con ecografías normales o con signos ecográficos de hematoma pequeños o dudodos y Rx de abdomen carente de imágenes sospechosas, p.ej., aumento significativo de la silueta renal, borramiento del psoas, desplazamiento colónico o diafragmático). En los enfermos con diagnóstico ecográfico claro o dudoso de hematoma asociado a síntomas no se procedió al alta hasta transcurridas $24 \mathrm{hrs}$. de absoluta estabilidad clínica ( ausencia de dolor, región lumbar y flanco no abombados ni contracturados, TA y FC normales), hematológica (con $\mathrm{Hb}$. y Hto. superiores a $10 \mathrm{mg} / \mathrm{dl}$ y $28 \%$ ) e imagenológica (diámetros y área del hematoma iguales o menores y seno renal normalmente visible).

El control evolutivo por vía telefónica fue realizado con la información de los pacientes (incluídos informes escritos de sus urólogos y/o estudios reali- zados ) durante un periodo postlitotricia comprendido entre los 7 y 19 meses (media $=13$ meses).

\section{RESULTADOS}

\section{a) Clasificación de los casos y frecuencias encontra- das:}

En las 324 litotricias consecutivas estudiadas se diagnosticaron un total de 42 hematomas (13\%) de diversa magnitud, de los cuales $37,(11,4 \%)$, fueron subcapsulares ("marginales o subserosos" e intrarrenales o "profundos"), 3 perirrenales puros, $(0,9 \%)$, y $2(0,6 \%)$, subcapsulares con derrame perirrenal. La ecografía fue no concluyente o dudosa en 26 casos ( $8 \%$ de las unidades renales tratadas), de estos finalmente fueron diagnósticados como portadores de hematoma dada su evolución dos pacientes, $(7,7 \%)$. (Figuras 1, 2 y 3 ).

De los 42 hematomas diagnosticados, 20 $(47,6 \%)$, se asociaron a algún síntoma probablemente atribuible al hematoma hasta el momento del alta ("hematomas síntomáticos: HS") lo que constituye el $6,2 \%$ de las unidades renales tratadas y $22(52,4$ $\%)$ fueron clasificados como "asintomáticos: $\mathrm{HA}^{\prime}, \mathrm{o}$ sea, el 6,8\% de las litotricias.

La incidencia acumulada de los hematomas que evolucionaron de forma progresiva ("hematomas progresivos: $\mathrm{HP}^{\prime \prime}$ ) fue del $2,16 \%$, o sea, 7 casos, lo que representa el $16,6 \%$ de los hematomas diagnósticados.

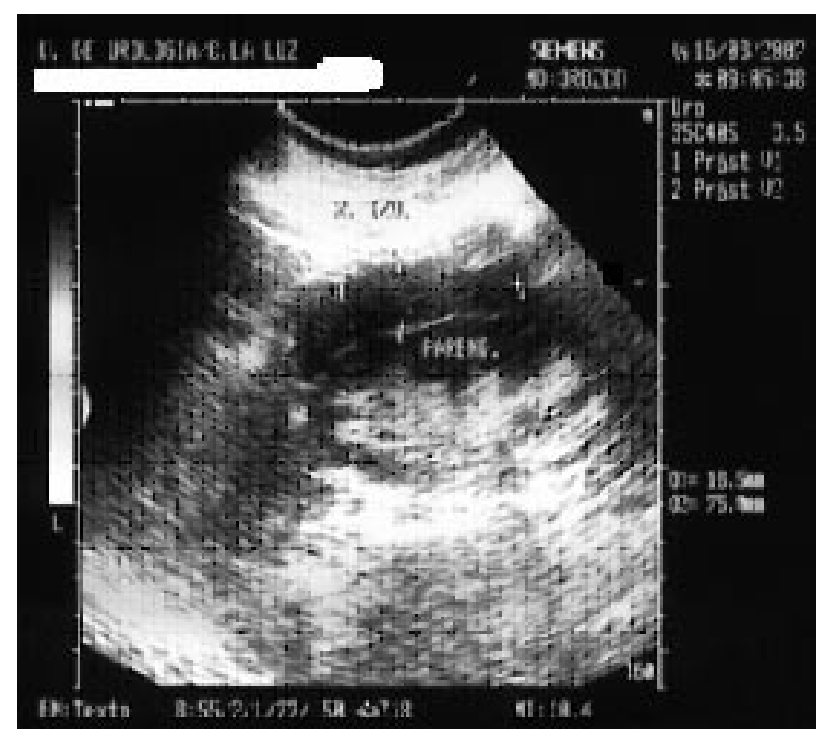

FIGURA 1. Hematoma perirrenal izquierdo. Corte longitudinal. 


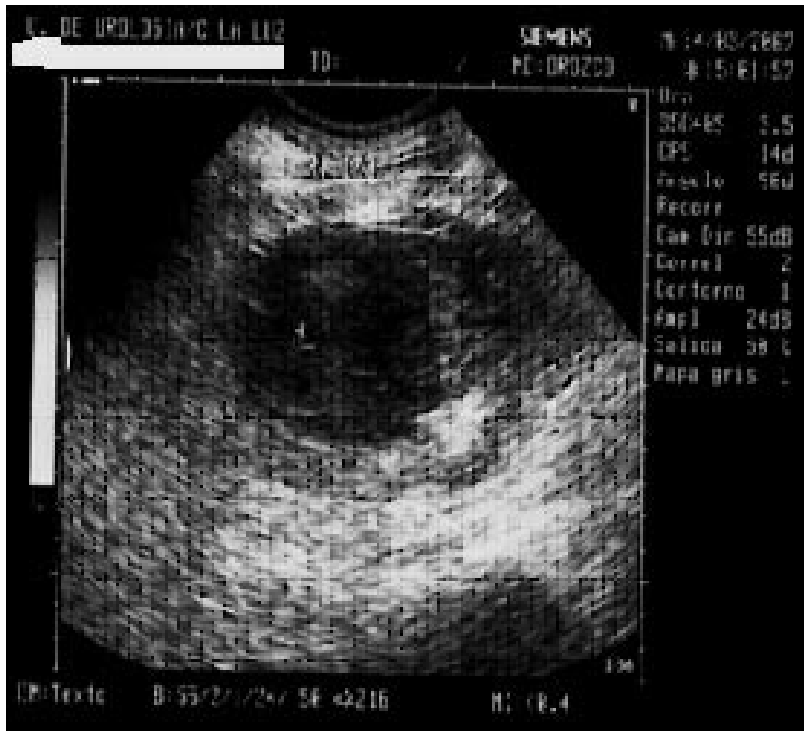

FIGURA 2. Hematoma subcapsular izquierdo con apertura perirrenal.

\section{b) Síntomas y signos:}

Constatamos relación estadísticamente significativa entre los hematomas sintomáticos y el mayor tamaño ("área eliptica") de estos ( $p<0,04)$ en comparación con los hematomas asintomáticos.

Los síntomas y signos observados en el grupo de HS fueron: dolor $(100 \%$ de los 20 hematomas síntomáticos), náuseas y vómitos (15\%), mareos e hipotensión arterial $(15 \%)$, palidez $(40 \%)$, gran hematoma subcutáneo $(0,3 \%)$ y oligoanuria $(0,3 \%)$, los dos últimos de aparición mas tardía (48 - 72 hrs.), por lo que podemos afirmar que el síntoma principal en estos casos es el dolor, el cual fué directamente

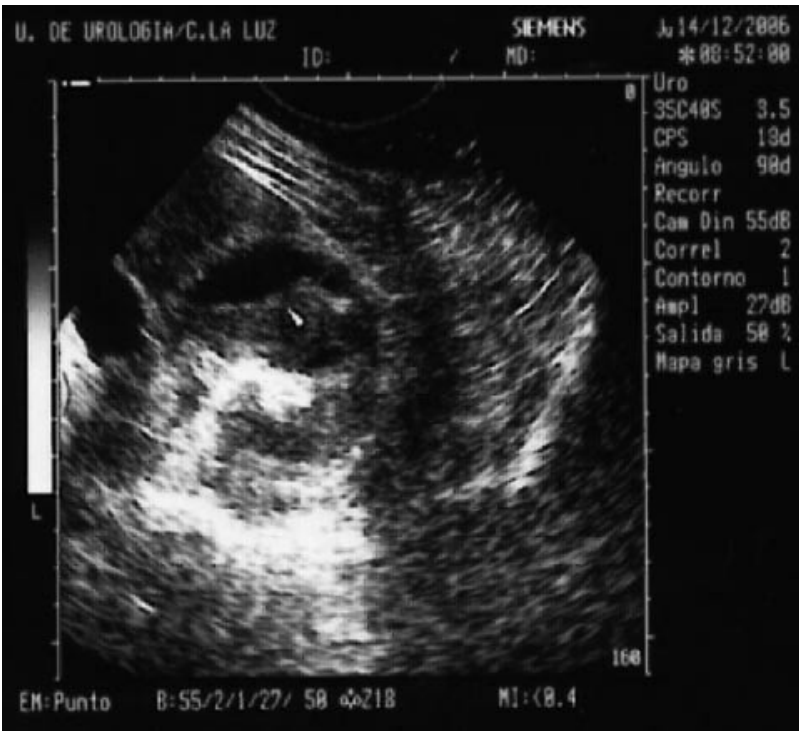

FIGURA 3. Hematoma subcapsular marginal derecho. Corte transversal.

proporcional, como se expone en el párrafo anterior, al volumen del hematoma; naturalmente si tenemos en cuenta los HA la frecuencia de dichos síntomas es inferior, pero aún así son significativas las diferencias encontradas, (Tabla I).

La palidez y las náuseas se relacionaron con la intensidad del dolor y con el tamaño del hematoma, $(p<0,05)$ pero no con la hipotensión arterial.

El dolor apareció entre las 4 y las 12 hrs posteriores a la litotricia, pero el $90 \%$ alcanzó su máxima intensidad entre las 4 y 8 hrs.

TABLA I. SÍNTOMAS Y SIGNOS EN PRESENCIA O AUSENCIA DE HEMATOMAS POSTLITOTRICIA.

\begin{tabular}{|l|c|c|c|c|}
\hline Síntomas y signos & \multicolumn{2}{|c|}{$\begin{array}{c}\text { Sin hematoma. N= 282 } \\
\text { No }\end{array}$} & \multicolumn{2}{c|}{$\begin{array}{c}\text { Con hematoma. N=42 } \\
\text { No }\end{array}$} \\
\hline Dolor intenso & 9 & 3,19 & 5 & $12^{*}$ \\
\hline Dolor moderado & 36 & 12,76 & 15 & $35,7^{*}$ \\
\hline Náuseas / vómitos & 9 & 3,19 & 3 & 7,1 \\
\hline Hipotensión arterial & 22 & 7,8 & 3 & 7,1 \\
\hline Pálidez & 10 & 3,54 & 8 & $19^{*}$ \\
\hline Hematoma subcutáneo severo & 0 & 0 & 1 & 2,4 \\
\hline
\end{tabular}

${ }^{\star}=p<0,05$ 
Las características semiológicas diferenciales entre los tipos de hematomas fueron: la intensidad, el tiempo de aparición y la localización del dolor; en los hematomas subcapsulares fue lumbar en el $95 \%$ y torácico en $5 \%$, (paciente con gran hemato$\mathrm{ma})$, mientras que en los perirrenales la tendencia es que aparezca mas tardíamente (entre las 6 y las 10 hrs.) con menor intensidad, de curso mas irregular y, en el $80 \%$ (4 pacientes), con irradiación o localización atípicas, estas fueron: hombro, infraescapular ipsilateral, precordial y flancoabdominal anterior difuso. La irritación del psoas fue mas infrecuente de lo esperado en los hematomas perirrenales (10\%), pero en relación con hematomas de gran tamaño. Estas características las resumimos en el Cuadro III.

Dos pacientes con hematomas del subgrupo HP resultaron inicialmente asintomáticos, o sea, el $28,6 \%$ del grupo HA.

La tipificación clínica completa de los hematomas diagnosticados se expone en la Tabla II.

\section{c) Evolución clínica, progresión de los hematomas y su diagnóstico:}

De los 42 hematomas dignosticados sólo 4 (9,5\% de los $\mathrm{H}$ y $20 \%$ de los HS) tuvieron signos ecográficos de progresión en las primeras 18 hrs., o sea, el $57,14 \%$ de HP; de los mismos 2 requirieron transfusión de hematíes concentrados sin otras medidas adicionales a la observación estricta, el reposo, la sueroterapia, antibioticoterápia y la analgesia. Otros 3 pacientes ( 2 con $\mathrm{HS}$ y 1 con $\mathrm{HA}$ ) evolucionaron progresivamente después de producirse el alta al cumplir con los criterios establecidos para la misma, así pues, finalmente la incidencia acumulada de hematomas progresivos resultó ser de 7 pacientes, 0 sea, el $2,16 \%$ de las unidades renales tratadas y el $16,66 \%$ de los hematomas.

Una paciente del grupo HP, a la que se le transfundió concentrados de hematíes y fué dada de alta por su estabilidad clínica y ecográfica durante 24 hrs., requirió posteriormente reingreso a los 5 días de la litotricia, ( 3 días después del alta), al acudir a urgencias por dolor intenso incontrolable, aunque no necesitó nuevas transfusiones a pesar de que según la TAC realizada padecía un hematoma de $12 \mathrm{~cm}$ de diámetro.

La progresión cuantitativa media ecográfica de los HP fué de $4 \mathrm{cms}$ para los diámetros y de 38,3 $\mathrm{cm} 2$ para el área eliptica., dicha progresión se produjo en los primeros 5 días si tenemos en cuenta los dos casos que fueron reingresados tras el alta inicial y no incluye los datos de 2 pacientes cuyo seguimiento se realizó en sus centros de procedencia sin haberse podido obtener los datos cuantitativos necesarios.

CUADRO III. TENDENCIAS CLIINICAS DIFERENCIALES ENCONTRADAS EN LOS HEMATOMAS POSTLITOTRICIA SINTOMÁTICOS $(\mathrm{N}=20)$.

\begin{tabular}{|l|l|l|}
\hline $\begin{array}{l}\text { Localización del dolor } \\
\text { e irradiación }\end{array}$ & $\begin{array}{l}\text { Intrarrenales/ subcapsulares } \\
\text { Lumbar (y torácico } \\
\text { si gran volumen) }\end{array}$ & $\begin{array}{l}\text { Pumboabdominal difuso, } \\
\text { hombro, precordial, e infraescapular. }\end{array}$ \\
\hline Intensidad del dolor & Intenso & Menos intenso \\
\hline Debut (tiempo) & $3-8$ hrs. & 6-10 hrs. \\
\hline $\begin{array}{l}\text { Signos de irritación } \\
\text { Psoica }\end{array}$ & No & Sí \\
\hline Curso del dolor & Constante & Irregular o insidioso \\
\hline Síntomas asociados & $\begin{array}{l}\text { Sudoración, palidez, } \\
\text { náuseas-vómitos, } \\
\text { mareos. }\end{array}$ & $\begin{array}{l}\text { Palidéz mas tardía, distensión } \\
\text { o malestar abdominal, mareos, } \\
\text { hematoma subcutáneo severo. }\end{array}$ \\
\hline Respuesta a analgésicos & Pobre & Mejor \\
\hline
\end{tabular}


TABLA II. CARACTERIZACIÓN DE LOS HEMATOMAS SEGÚN LAS UDS. RENALES TRATADAS.

\begin{tabular}{|l|c|}
\hline \multicolumn{1}{|c|}{ Características } & No. de casos (\%) N = 324 \\
\hline Total de hematomas & $42(13 \%)$ \\
\hline Hematomas asintomáticos & $22(6,8 \%)$ \\
\hline Hematomas sintomáticos & $20(6,2 \%)$ \\
\hline Hematomas progresivos antes de 24 hrs. & $4(1,23 \%)$ \\
\hline Hematomas progresivos después de 24 hrs. & $3(0,93 \%)$ \\
\hline Hematomas en riñón derecho & $16(4,9 \%)$ \\
\hline Hematomas en riñón izquierdo & $27(8,3 \%)$ \\
\hline Hematomas progresivos con inestabilidad hemodinámica & $2(0,6 \%)$ \\
\hline Hematomas intrarrenales o subcapsulares & $37(1,4 \%)$ \\
\hline Hematomas subcapsulares con apertura perirrenal & $2(0,61 \%)$ \\
\hline Hematomas perirrenales & $3(0,92 \%)$ \\
\hline Hematomas en 1/3 superior & $7(2,16 \%)$ \\
\hline Hematomas en 1/3 medio & $19(5,86 \%)$ \\
\hline Hematomas en 1/3 inferior & $14(4,3 \%)$ \\
\hline Hematomas de localización indefinida o difusos & $2(0,74 \%)$ \\
\hline Area elíptica inicial de los hematomas (media y DS. / cm2) & $8+/-6,2$ \\
\hline Diámetros iniciales de los hematomas (media y DS / cms.) & $3,8 \times 2,5+/-1,54 \times 1,14$ \\
\hline Hematomas con volumen inicial igual o superior a $1 / 3$ del riñón & $8(2,46 \%)$ \\
\hline
\end{tabular}

En uno de los pacientes con HP la imagen ecográfica inicial era dudosa y el diagnóstico fue asumido teniendo en cuenta el incremento dimensional progresivo renal con "expansión" del parénquima y rechazo del seno renal, expresado por una imagen que identificamos como "signo de la media luna sinusal" (Figura 4), así como la presencia de dolor atípico infraescapular intenso, no obstante, el leve descenso de la $\mathrm{Hb}$ y el Hto. y su estabilidad hemodinámica evitaron la necesidad de transfusión.

El $42,8 \%$ (3 pacientes) de los HP no fueron clasificados como tales en las primeras $24 \mathrm{hrs}$. por no manifestarse ecográfica ni clínicamente como tal, incluso uno de estos $(14,3 \%)$ no presentó síntomas en dicho periodo de tiempo, se trató de una paciente cuadriplégica con un dudoso y pequeño hematoma en la ecografía inicial, que reingresó a las 48 hrs. con importante hematoma subcapsular y leve perirrenal, descenso de $\mathrm{Hb}$ y Hto. $(6,1 \mathrm{mg} / \mathrm{dl}$ y $17,6 \%)$,

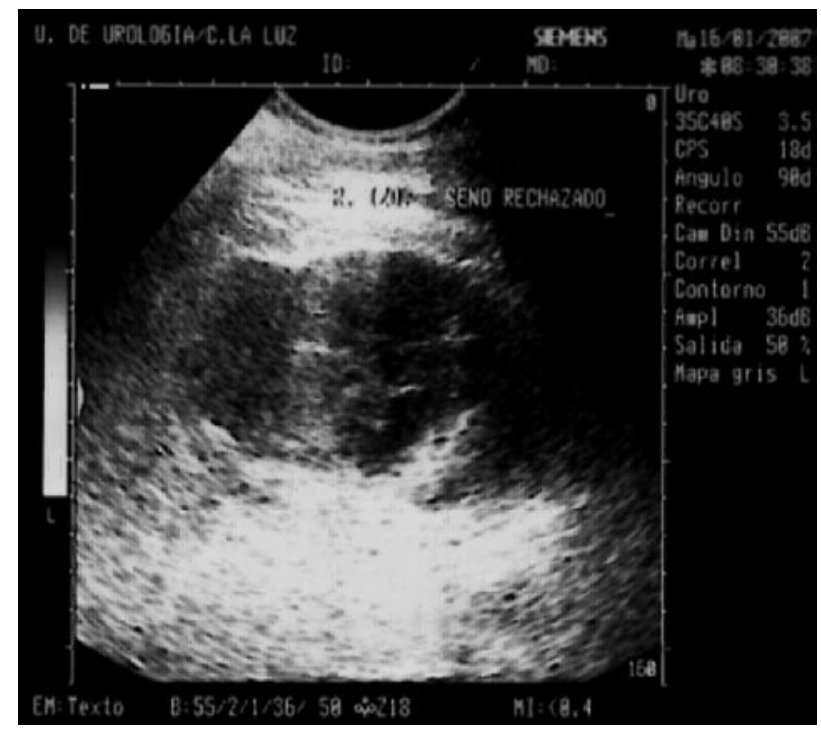

FIGURA 4. Seno renal rechazado con aspecto de "media luna". 


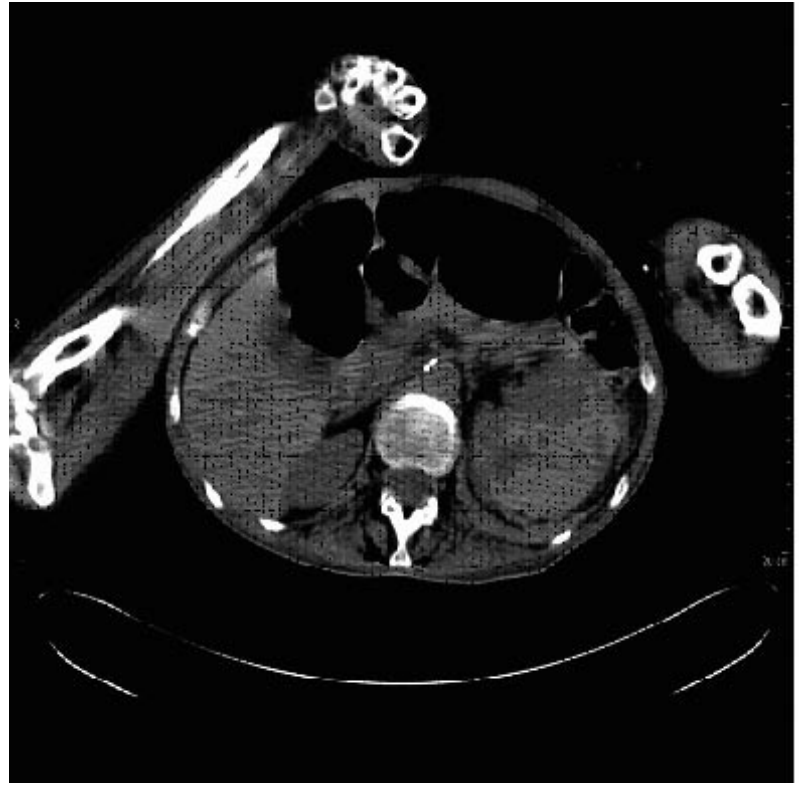

FIGURA 5. Hematoma subcapsular izquierdo con apertura perirrenal. Pte. cuadriplégica asintomática a las 24 hrs. y reingresó a las 48 hrs.

fiebre, oligoanuria y palidez cutáneomucosa, (Figura 5), otro paciente presentó un hematoma sintomático de evolución ecográfica estable y remisión sintomática por $24 \mathrm{hrs}$. teniendo que ser atendido en los días ulteriores al alta, en su centro de procedencia, por dolor lumbar recurrente e intenso y gran hematoma subcutáneo. En consecuencia, la ecografía fué dudosa inicialmente en 2 casos, o sea, el 28,57\% de los pacientes que presentaron evolución progresiva del hematoma.

Tras contactar telefónicamente con los pacientes que presentaron hematomas tras un internalo postlitotricia comprendido entre los 7 y 19 meses comprobamos que 5 enfermos, $111,9 \%$ de dichos pacientes y 1,5\% de las litotricias), evolucionaron con dolor lumbar o lumboabdominal entre los 2 y 6 meses posteriores a la litotricia, uno de estos pacientes aqueja un dolor que aumenta a la presión del decúbito y ante la inspiración.

\section{d) Variables analizadas como posibles factores pre- disponentes o condicionantes:}

Los factores de riesgo o predisponentes asociados a la aparición de hematomas postlitotricia señalados por los investigadores en la literatura y los resultados del análisis estadístico realizado en el presente estudio, que detallaremos a continuación, se resumen comparativamente en el Cuadro II.

- Número de litiasis: no se observó que este se correspondiera directa y proporcionalmente con la aparición postoperatoria de hematomas según el test de regresión logística, pero si hubo diferencias significativas entre los pacientes con litiasis única y los portadores de dos o mas cálculos, con una incidencia mayor para este último subgrupo $(P<0,05)$.

\section{- Localización y multiplicidad litiásica en relación con el sitio del hematoma y la sintomatología: se constató que el $93 \%$ de los pacientes con hematoma de $1 / 3$ medio o inferior tenían cálculos piélicos, en cáliz in- ferior o uréter proximal, mientras que el $89 \%$ de los hematomas de $1 / 3$ superior tenían litiasis piélica y en caliz superior única o múltiple incluídas ambas localizaciones (Tabla III). Para los casos con litiasis renal la aparición de hematomas ocurrió en el 14 $\%$, mientras que para los cálculos situados en UPU o ureterales próximos a esta lo hizo en el 7,8 \% y en los que abarcaron ambas localizaciones, 12,5\% (Tabla IV).}

En cuanto a la sintomatología del hematoma y su relación con la situación del calculo tratado es relevante el hecho de que para los HS la ubicación calicial predominó significativamente mientras que en las litiasis piélicas únicas los hematomas fueron asintomáticos en el 91 \% (10 de 11 casos) (Gráfico 1).

En el caso de los HP existió un predominio del lado izquierdo al igual que sucedió con la loca-

TABLA III. LOCALIZACIÓN Y NÚMERO DE LITIASIS vS SITIO DE HEMATOMA ( $n=42$ ).

\begin{tabular}{|l|c|c|}
\hline Sitio del hematoma & Litiasis pielica, cáliz inferior o ureter proximal & Litiasis piélica y cáliz superior o múltiple. \\
\hline $1 / 3$ medio o inferior & $93 \%$ & $11 \%$ \\
\hline $1 / 3$ superior & $7 \%$ & $89 \%$ \\
\hline
\end{tabular}




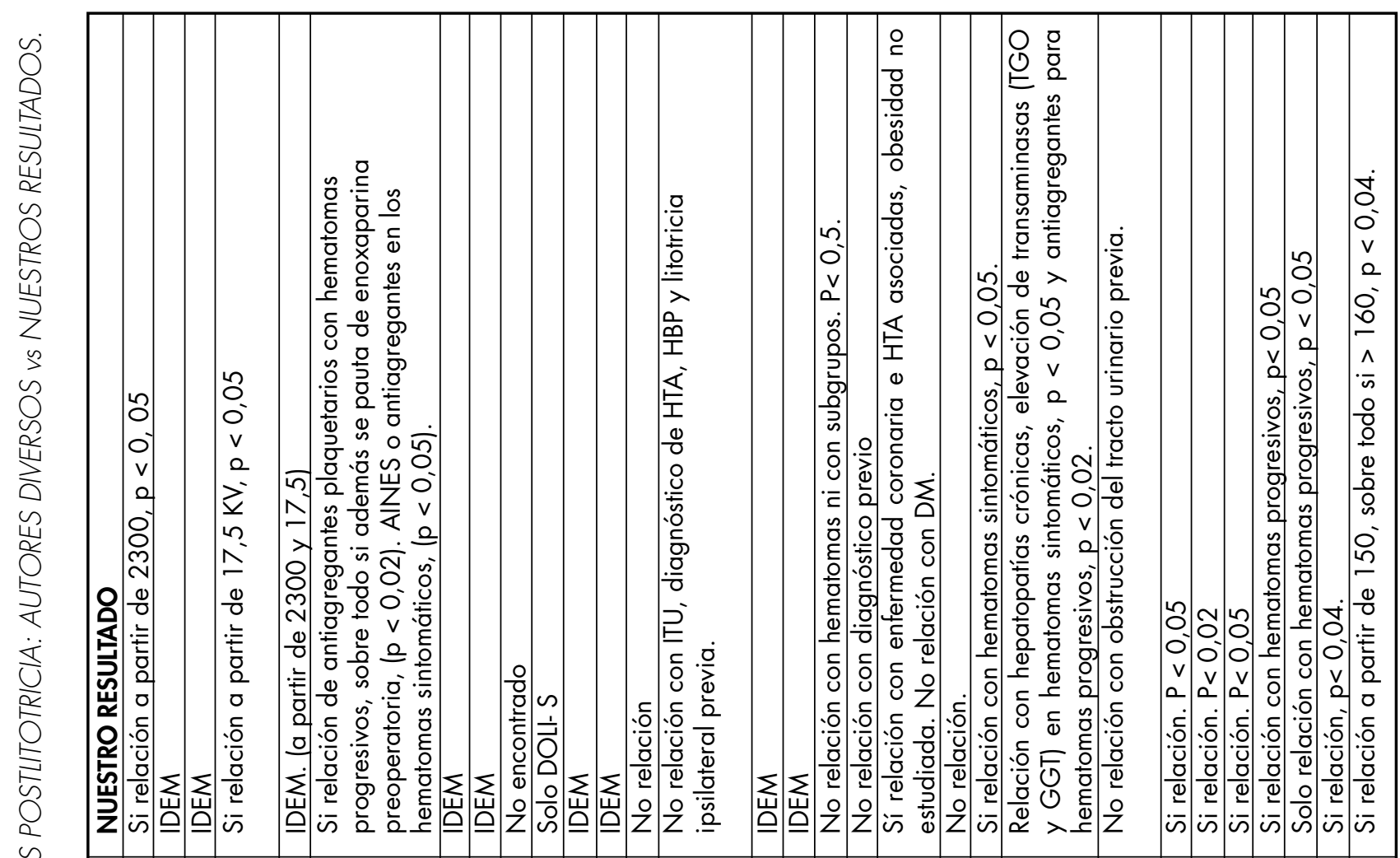

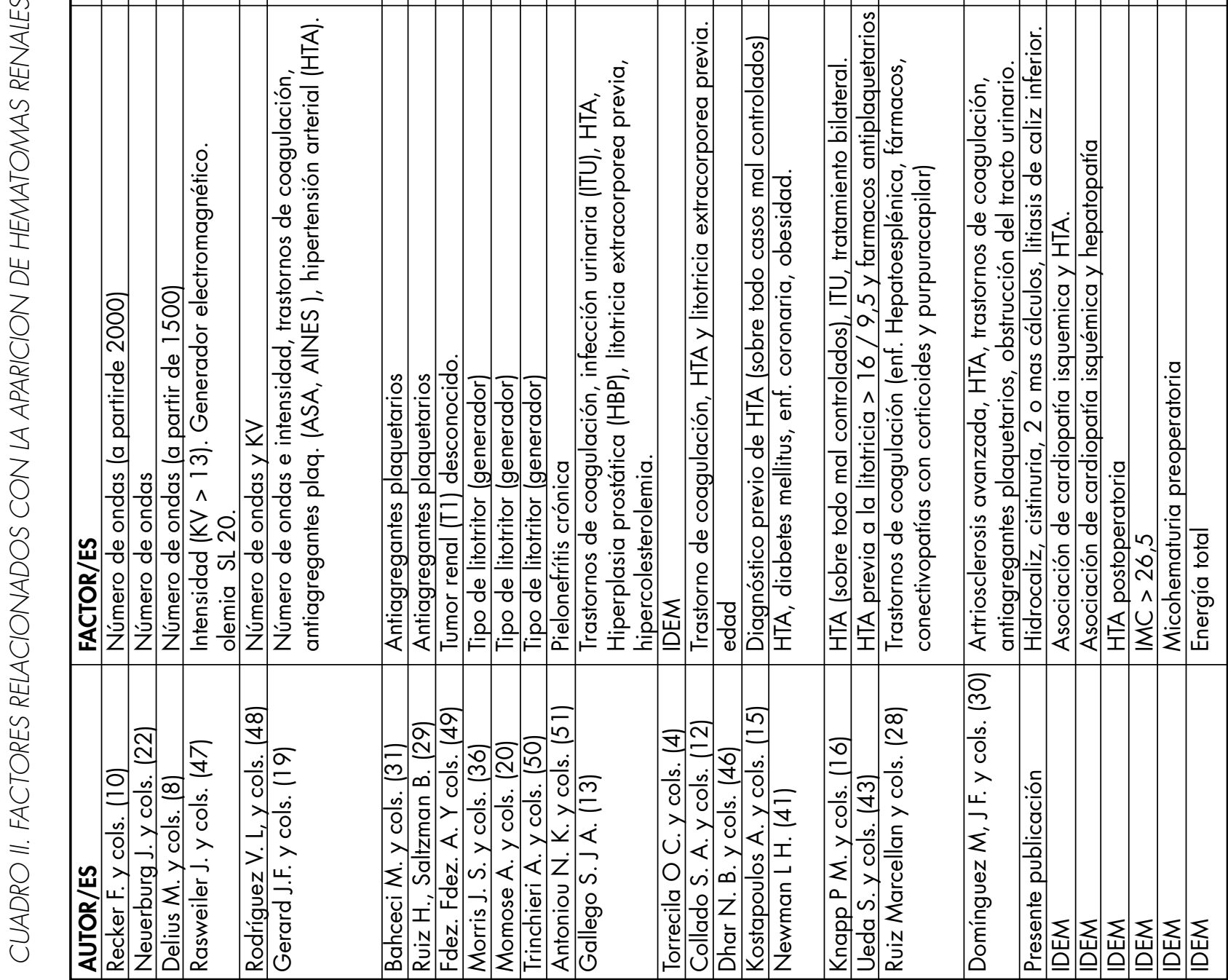




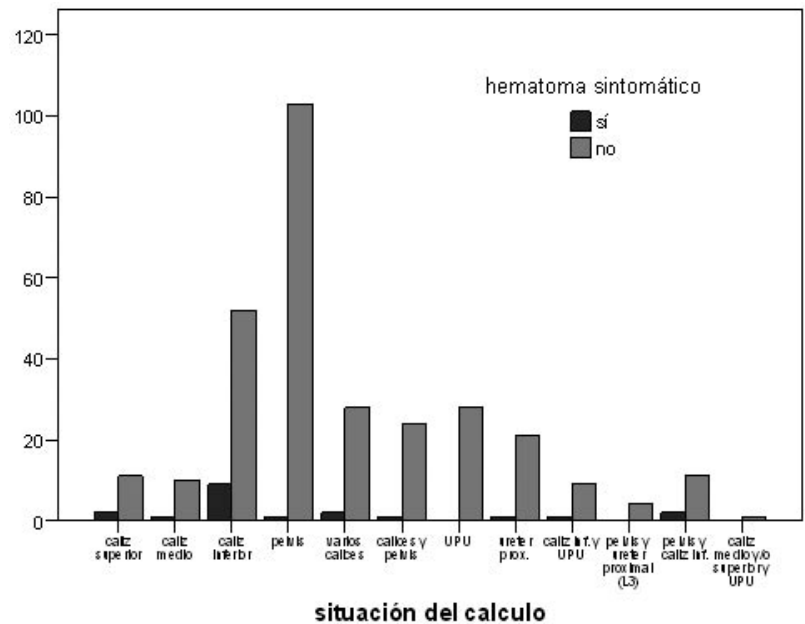

Gráfico 1: RELACIÓN ENTRE SITUACIÓN DEL CÁLCULO E INCIDENCIA DE HEMATOMAS SINTOMÁTICOS. $N=324$.

lización del cálculo en el cáliz inferior en 6 de los 7 pacientes $(85,7 \%)$, así mismo en el grupo de HS el 55\% (11 hematomas), eran casos portadores de litiasis en cáliz inferior, ( $p<0,05)$, en los Gráficos 1 y 2 se puede observar la incidencia y proporción de hematomas (HS y HP), según la distribución de las diversas localizaciones de los cálculos tratados.

El predominio de la localización calicial para los hematomas en general (grupo H) también se ilus- tra en el Gráfico 3; en dicho grupo no observamos relación estadísticamente significativa con la unidad renal tratada (izquierda o derecha).

- Sexo y antecedente de LEOCH ipsilateral: no se constató relación estadísticamente significativa en ambos a pesar de que la LEOCH ipsilateral previa ha sido sugerida por otros (12).

- Enfermedad renal asociada: globalmente, en los 95 pacientes con enfermedad renal previa, no fue posible definir las relaciones estadísticas con la aparición de hematoma postlitotricia por la dispersión de los datos (entidades nosológicas) aunque cabe señalar que, al individualizar las afecciones, la presencia de litiasis en hidrocaliz o de cistina se correspondió con el diagnóstico de hematoma en el $40 \%$ y $66,6 \%$ respectivamente, existiendo relación estadísticamente significativa con ambos $(p<0,05)$. La ITU recurrente estuvo presente en el $24 \%$ de los casos pero ni esta, ni la uropatía obstructiva, ni el grado de esta última previo a la litotricia, demostraron asociación con los hematomas en general ni con los subgrupos establecidos ("síntomático", "asintomáticos"y"progresivos").

- Dilatación pielocalicial postlitotricia: se observó relación en el grupo de los HS $(p<0,05)$, este dato será comentado en la discusión al abordar los síntomas y signos.

- Enfermedades asociadas, (no renales): En los 152 pacientes que tenían enfermedades no renales aso-

TABLA IV. FRECUENCIA DE HEMATOMAS SEGÚN LOCALIZACIÓN DE LA/S LITIASIS.

\begin{tabular}{|l|c|c|c|}
\hline Localización de litiasis & Renal. N= 257 & UPU / uréter prox. N= 51 & Renal y UPU. N= 16 \\
\hline No. y \% de hematomas & $36(14 \%)$ & $4(7,8 \%)$ & $2(12,5 \%)$ \\
\hline
\end{tabular}

TABLA V. INCIDENCIA DE LOS HEMATOMAS EN PACIENTES CON DIAGNÓSTICO PREVIO DE HTA AISLADO vs. ASOCIADO A CARDIOPATÍA ISQUÉMICA O DIABETES MELLITUS.

\begin{tabular}{|c|c|c|c|c|}
\hline $\begin{array}{l}\text { HTA +/_ Cardiopatía } \\
\text { isquémica o Diabetes } \\
\text { Mellitus } \\
\text { HTA solo. } \mathrm{N}=80\end{array}$ & $\begin{array}{c}\text { Hematomas } \\
\text { (total) } \\
\text { Ptes. y \% } \\
8(10 \%)\end{array}$ & $\begin{array}{c}\text { Hematomas } \\
\text { sintomáticos } \\
\text { Ptes. y \% } \\
3(3,75 \%)\end{array}$ & $\begin{array}{c}\text { Hematomas } \\
\text { asintomáticos } \\
\text { Ptes. y \% } \\
4(5 \%)\end{array}$ & $\begin{array}{c}\text { Hematomas } \\
\text { progresivos } \\
\text { Ptes. y \% } \\
1(1,25 \%)\end{array}$ \\
\hline HTA y Card. Isq. $N=9$ & $4(44,4 \%)^{*}$ & $2(22,2 \%)$ & $2(22,2 \%)$ & $0(0 \%)$ \\
\hline HTA y Diab. $M . N=13$ & $1(7,7 \%)$ & $1(7,7 \%)$ & $0(0 \%)$ & $0(0 \%)$ \\
\hline
\end{tabular}

${ }^{\star}=p<0,05$ 


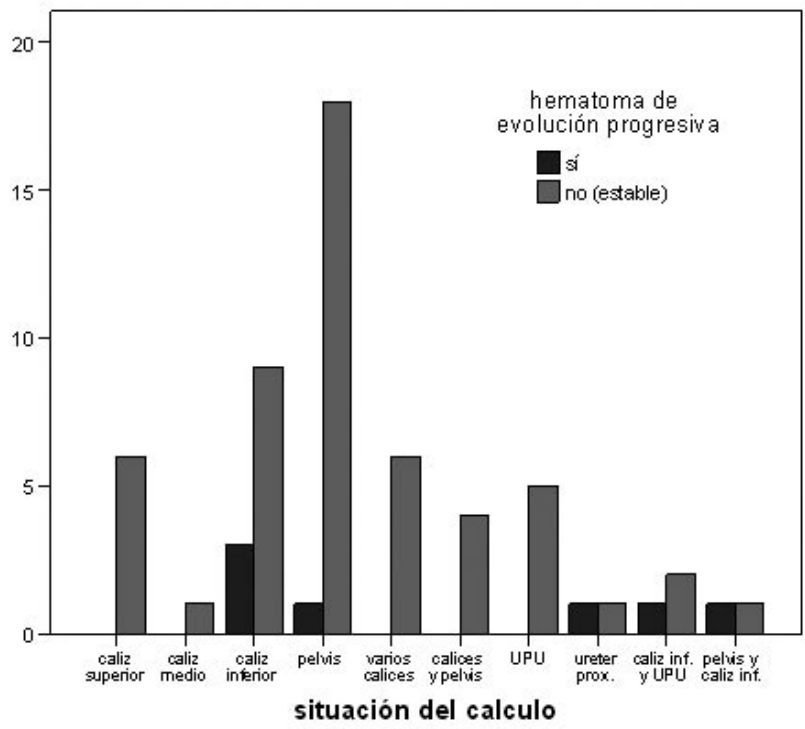

Gráfico 2: RELACIÓN ENTRE SITUACIÓN DEL CÁLCULO Y LA INCIDENCIA DE HEMATOMAS DE EVOLUCIÓN PROGRESIVA EN LOS CASOS CON ECOGRAFÍAS INICIALES CLARAS O SOSPECHOSAS DE HEMATOMAS. $N=66$.

ciadas la presencia de estas, en general, no influyó en la incidencia de hematomas: 11,2\% (17 casos) en pacientes con antecedentes y 13,3\% (23 casos) en los pacientes sin antecedentes patológicos no urinarios. Al analizar individualmente las patologías asociadas no observamos relación estadística con la aparición de hematomas en los pacientes con diagnóstico previo de HTA, (Tabla V); sólo resultó significativa la asociación de las hepatopatías crónicas (Hepatitis B y Cirrosis) en el grupo HS ( $p<0,05)$.

Analizamos las diversas asociaciones entre entidades nosológicas concomitantes y resultaron relacionadas significativamente la asociación de cardiopatía isquémica con HTA para los hematomas en general y la de hepatopatías con cardiopatía isquémica para dicho grupo y para los $\mathrm{HS}(P=0,05)$. La asociación de Diabetes Mellitus con cardiopatía isquémica o HTA no demostró relación estadística con los hematomas para ningún grupo (Tabla $\mathrm{V}$ ).

- Antiagregantes plaquetarios y anticoagulantes: No se constató relación suficientemente significativa entre el uso de antiagregantes, (en general, ni por principio activo), suspendidos al menos 5 días antes de la litotricia y el grupo $\mathrm{H}$, ni con los subgrupos $\mathrm{HS}$ y HA, pero sí en el grupo de los HP cuando dicha progresión ocurre en las primeras $18 \mathrm{hrs}$. $(\mathrm{p}=0,02)$, relación que se pierde cuando se incluyen los hema-

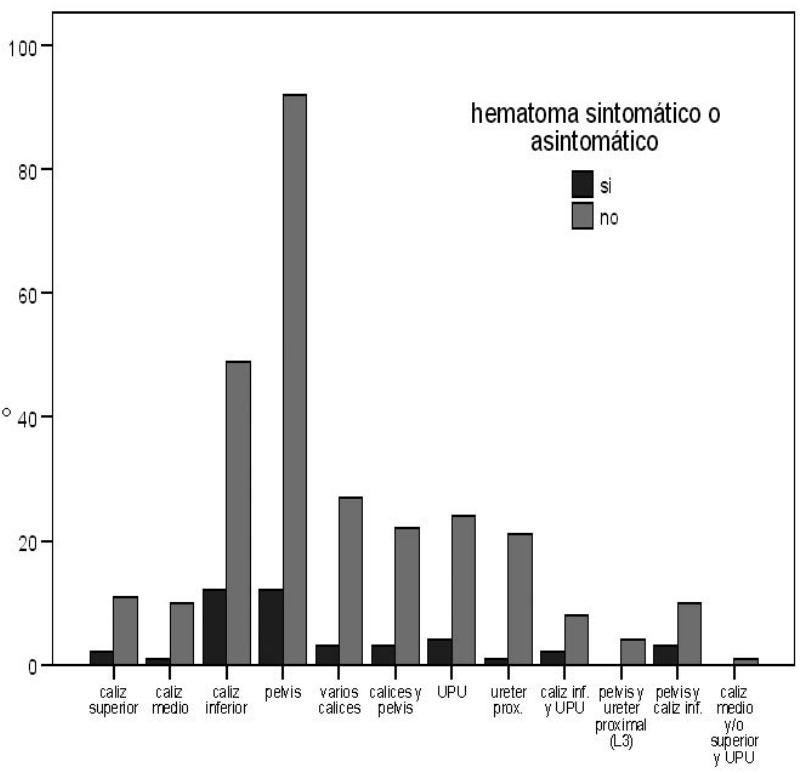

situación del calculo

\section{Gráfico 3: RELACIÓN ENTRE SITUACIÓN DEL CÁLCULO Y TOTAL DE HEMATOMAS DIAGNÓSTICADOS, ISINTOMÁTICOS O NOI. $N=324$.}

tomas cuya progresión se evidenció después de las 48 hrs. ( $p=0,2$ ), por otra parte cuando por condiciones particulares del paciente su médico no consideró oportuno suspender totalmente el tratamiento antiagregante y se indicó pauta de "solapamiento" de este con enoxoparina se comprobó relación estadística significativa con los HS ( $p<0,05)$ y HP ( $p<$ $0,02)$. Un dato a destacar es el hecho de que los tres pacientes consumidores habituales de ibuprofeno y piroxican de manera prolongada presentaron hematoma postlitotricia; si incluímos estos en el conjunto de pacientes con medicación antiagragegante teniendo en cuenta el potencial de estos en este sentido (ver discusión) si se observa relación estadística con los pacientes del grupo HS, ( $p<0,05)$ (Tabla VI).

- Índice de masa corporal (IMC): No se encontró relación de los hematomas con el incremento progresivo del IMC, no obstante, en el grupo de HP se comprobó que $75 \%$ tenían un IMC mayor de 26,5.

- Tensión arterial perioperatoria: no observamos relación estadística de los hematomas con el comportamiento de la TA perioperatoria en términos generales (gupo H); sin embargo, si la hubo con la HTA preoperatoria para el grupo HS (Gráfico 4), la incidencia de estos aumentó proporcionalmente de acuerdo con el grado de hipertensión, (Gráfico 5); así mismo, también se observó correlación significativa, (Spearman 


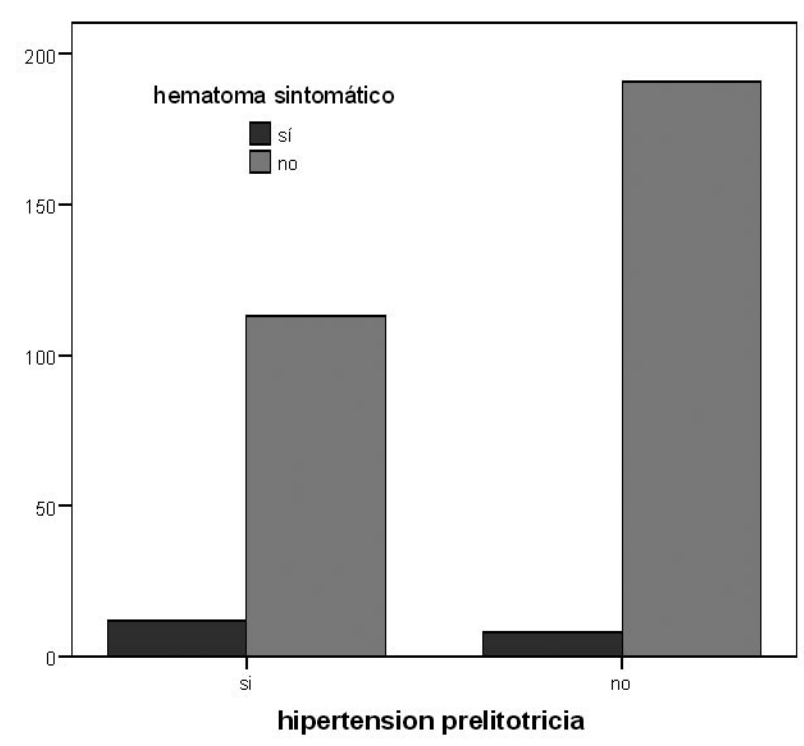

Gráfico 4: RELACIÓN ENTRE LA HTA PREVIA A LA LITOTRICIA Y LA INCIDENCIA DE HEMATOMAS SINTOMÁTICOS: $N=324$.

$\alpha=0,05)$ y regresión logística $(p<0,05)$ entre los HP y la HTA postoperatoria (en las primeras $6 \mathrm{hrs}$. del postoperatorio) tanto para los hematomas diagnosticados como para los casos con ecografías iniciales no concluyentes, (Gráfico 6).

La HTA perioperatoria no se relacionó con el volumen inicial del hematoma. La HTA perioperatoria

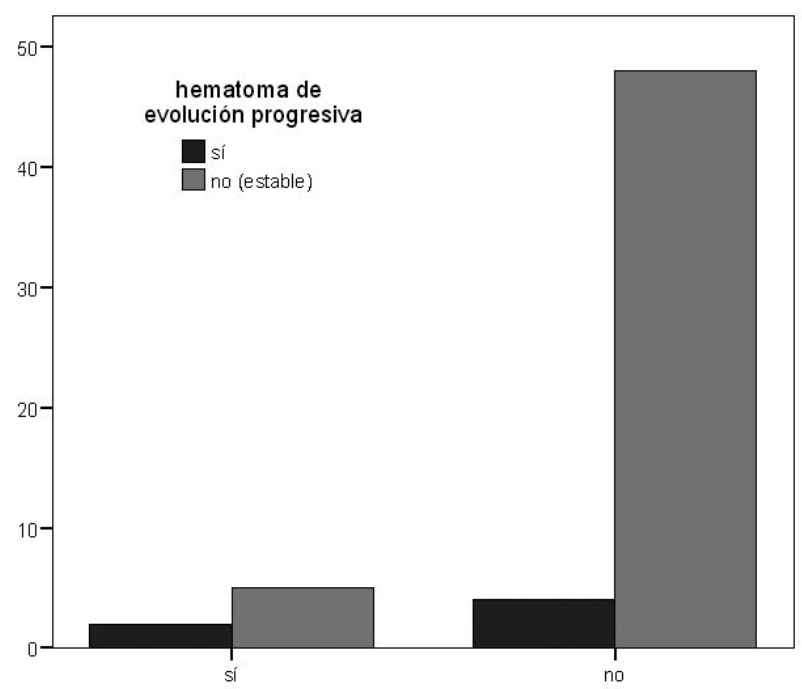

cifras de TA postop. super. a 13/8,5

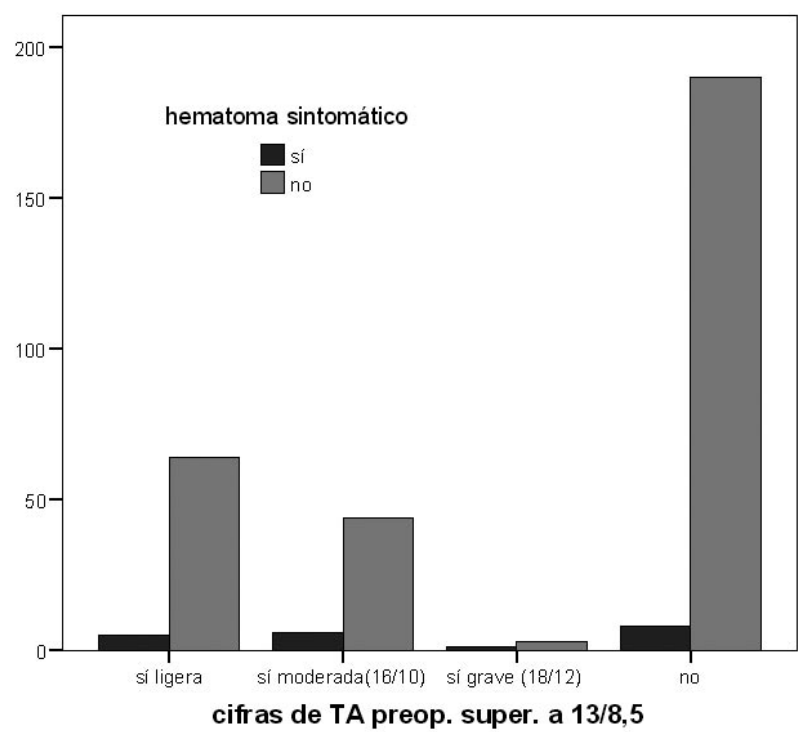

Gráfico 5: RELACIÓN ENTRE LA HTA PREVIA A LA LITOTRICIA Y LA INCIDENCIA DE HEMATOMAS SINTOMÁTICOS SEGÚN EL GRADO DE HIPERTENSIÓN. $N=324$.

como cofactor en el análisis estadístico de pacientes con tratamiento antiagregante plaquetario sólo resultó asociado a los HP y únicamente cuando se pautó enoxoparina al suspenderse el antiagregante, $p<$ 0.05 .

- Tamaño inicial del hematoma: El 85,7\% de los HP tuvo ambos diámetros iniciales de 40 mas, $(p<0,06)$.

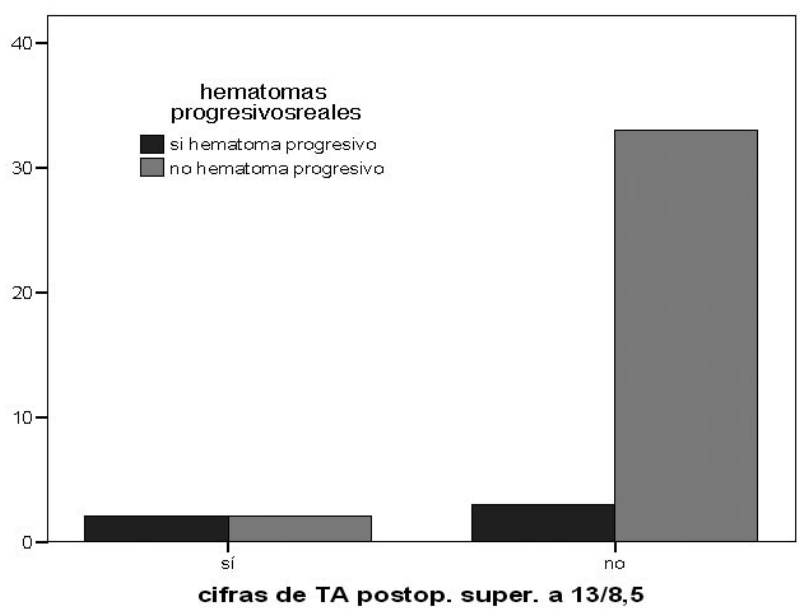

Gráfico 6: RELACIÓN DE LA HTA POSTERIOR A LA LITOTRICIA CON HEMATOMAS PROGRESIVOS: A) EN PACIENTES CON CAMBIOS ECOGRÁFICOS POSTLITOTRICIA CLAROS O DUDOSOS DE HEMATOMA. $N=66$.

B) EN PACIENTES FINALMENTE CLASIFICADOS PORTADORES DE HEMATOMA. $N=42$. 


\section{TABLA VI. RELACIÓN DE FÁRMACOS ANTIAGREGANTES; ANTICOAGULANTES; AINES, Y OTROS POTENCIALMENTE HEPATOTÓXICOS; CON LA INCIDENCIA DE HEMATOMAS VS NO CONSUMIDORES DE LOS MISMOS.}

\begin{tabular}{|c|c|c|c|c|c|c|}
\hline $\begin{array}{l}\text { Clasificación } \\
\text { por consumo } \\
\text { de fármacos } \\
\text { Clasificación } \\
\text { de hematomas }\end{array}$ & $\begin{array}{c}\begin{array}{c}\text { Antiagregantes } \\
\text { plaquetarios }\end{array} \\
\mathrm{N}=\mathbf{2 4}\end{array}$ & \begin{tabular}{|c} 
AINES \\
(consumidores \\
importantes) \\
$\mathrm{N}=4$
\end{tabular} & $\begin{array}{l}\text { Antiagregantes } \\
\text { + enoxoparina }\end{array}$ & $\begin{array}{c}\text { Antiagregantes } \\
\text { ○ AINES } \\
\text { N= 28 }\end{array}$ & \begin{tabular}{|c|}
$\begin{array}{c}\text { Otros } \\
\text { (potenciales } \\
\text { hepatotóxicos) }\end{array}$ \\
$\mathrm{N}=11$
\end{tabular} & \begin{tabular}{|c} 
Pacientes no \\
consumidores \\
de los fármacos \\
analizados \\
$\mathrm{N}=\mathbf{2 8 5}$
\end{tabular} \\
\hline $\begin{array}{l}\text { Hematoma } \\
\text { sintomático }\end{array}$ & $3(12,5 \%)^{*}$ & $2(50 \%)^{* * *}$ & $3(75 \%)^{* * *}$ & $5(17,9 \%)^{* *}$ & $2(18,2 \%)$ & $14(4,9 \%)$ \\
\hline $\begin{array}{l}\text { Hematoma } \\
\text { asintomático }\end{array}$ & $3(12,5 \%)^{*}$ & $1(25 \%)^{* *}$ & 0 & $4(14,3 \%)$ & 0 & $18(6,3 \%)$ \\
\hline $\begin{array}{l}\text { Hematoma } \\
\text { progresivo }\end{array}$ & $1(4,2 \%)^{* * *}$ & 0 & $3(75 \%)^{* * *}$ & $1(3,6 \%)^{* *}$ & 0 & $3(1 \%)$ \\
\hline $\begin{array}{l}\text { Total de } \\
\text { hematomas }\end{array}$ & $7(29,2 \%)^{* *}$ & $3(75 \%)^{* * *}$ & $3(75 \%)^{* * *}$ & $9(33,3 \%)^{* *}$ & $2(18,2 \%)$ & $34(11,9 \%)$ \\
\hline
\end{tabular}

† $75 \%$ fueron sintomáticos progresivos.

$$
{ }^{*} p<0,09 * * p<0,05 \quad * * * p<0,02
$$

- Parámetros de laboratorio: el 61,9\% ( 26 pacientes) de los enfermos que presentaron hematoma tenían microhematuria preoperatoria resultando estadísticamente significativa dicha asociación $(p<0,04)$. No observamos relación estadística entre los hematomas en general (ni con los subgrupos establecidos) y los valores preoperatorios de $\mathrm{Hb}$, Hto., creatinina, INR, fibrinogeno, plaquetas, ni con la TGP; sin embargo, si se evidenció entre la elevación de la TGO y la GGT y la aparición de los hematomas o grupo H (P $<0,05)$.

Respecto al monitoreo de la $\mathrm{Hb}$ y el Hto. en los pacientes con hematomas sintomáticos se observó un descenso medio de $3 \mathrm{mg} / \mathrm{dl}$ y $17 \%$ respectiva- mente, (Tabla VII), el descenso de estos parámetros no se asoció a la inestabilidad hemodinámica.

- Parámetros terapéuticos de la litotricia: no constatamos evidencias de asociación para valores crecientes en número de impactos $u$ ondas, intensidad y energía total aplicados, sin embargo, al estratificar las muestras, se observó diferencias significativas $(P=0,04)$ cuando la energía total es mayor o menor de 150, (Tabla VIII), siendo mayor la aparición de hematomas cuando esta supera dicha cifra, particularmente para los HP cuando es mayor o igual a 160 ( $p<0,03)$, siendo la probabilidad de hematomas 1,6 veces mayor; de igual manera, observamos que para cifras mayores de 2300 impactos se evidenció asociación

TABLA VII. DESCENSO DE Hb. Y Hto. Y RESPUESTA POSTRANSFUSIONAL EN HEMATOMAS PROGRESIVOS.

\begin{tabular}{|l|c|c|c|c|c|}
\hline $\begin{array}{l}\text { Parámetro } \\
\text { hemático }\end{array}$ & Media y DS & Máximo & Mínimo & $\begin{array}{c}\text { Descenso medio } \\
\text { según valor } \\
\text { preoperatorio }\end{array}$ & $\begin{array}{c}\text { Valores posteriores a } \\
\text { transfusión (3 a 5 uds.) }\end{array}$ \\
\hline Hb (mg/dl) & $9,96+/-2$ & 13,2 & 6,1 & 3 & $10,3+/-2,9$ \\
\hline Hto.(\%) & $28,6+/-5,7$ & 37,7 & 17,6 & 17 & $29,3+/-8$ \\
\hline
\end{tabular}


TABLA VIII. INCIDENCIA DE HEMATOMA POSTLITOTRICIA SEGÚN LA DOSIS DE ENERGIA TOTAL. N = 314.

\begin{tabular}{|l|l|l|c|}
\hline Energía total & Hematoma & No hematoma & Total \\
\hline 0 a 150 & $6^{*}$ & 94 & 100 \\
\hline 150 a 300 & $33^{*}$ & 162 & 195 \\
\hline Mas de 300 & $3^{*}$ & 16 & 19 \\
\hline Total & 42 & 272 & $314^{* *}$ \\
\hline
\end{tabular}

* $p<0,05 \quad * * 10$ casos sin el dato.

con los grupos $\mathrm{H}$ y $\mathrm{HS}(\mathrm{p}<0,05)$, dato que no se reprodujo en los HA, (Tabla IX). La intensidad fue relativamente significativa cuando fue mayor de $17 \mathrm{KV}$ para los hematomas en general y los HP, ( $p<0,07)$; la frecuencia no fue un dato estadísticamente valorable en nuestros pacientes (ver discusión).

\section{e) Manejo terapéutico transfusional:}

Sólo tres de los 42 hematomas necesitaron transfusión de concentrado de hematíes, dos resultaron HS, uno HA (en las primeras 24 hrs.) y sólo dos (uno de cada grupo citado) fueron clásificados como HP; por tanto, dichas transfusiones representaron: el 0,9\% de las litotricias realizadas, 7,1\% de todos los hematomas diagnosticados, $10 \%$ de los $\mathrm{HS}, 4,5 \%$ de los $\mathrm{HA}$ y $28,6 \%$ de los $\mathrm{HP}$, aunque para este último grupo cabe señalar que necesitaron transfusión el $50 \%$ de aquellos que progresaron en las primeras $24 \mathrm{hrs}$. La recuperación postransfusional de la $\mathrm{Hb}$ y el Hto. fue discreta, (Tabla VIII). La cantidad de concentrado de hematíes necesaria fue de 3 a 5 unidades.

\section{DISCUSIÓN}

Comentaremos solamente los aspectos de mayor interés en virtud de los resultados.

- Incidencia y prevalencia: La prevalencia o la incidencia de los hematomas renales y perirrenales reportada en la literatura urológica varía ampliamente desde 0 al $60 \%$ lo cual creemos que está en relacionado con los criterios estadísticos y clínicos seguidos por los diferentes autores y el tipo de estudio realizado (experimental, observacional prospectivo o retrospectivo). Muchos artículos con series amplias sólo hacen mención de la incidencia de hematomas sintomáticos o aquellos que requirieron tratamiento específico, presentando cifras que no superan el 1,2\% (2-4, 12, 14-16), sin embargo los estudios con menor número de casos pero que han tenido por objetivo específico el diagnóstico de los hematomas con métodos imagenológicos mas sensibles como la TAC y la $\mathrm{RMN}$ son los que mayores incidencias reportan, con valores del 24 al 60\% (17-19); otro aspecto a tener en cuenta para la valoración correcta de los datos

TABLA IX. INCIDENCIA DE HEMATOMA POSTLITOTRICIA SEGÚN NÚMERO DE ONDAS. N = 323.

\begin{tabular}{|l|l|l|l|}
\hline No. de ondas & Con hematoma & Sin hematoma & Total \\
\hline Hasta 2300 & $9^{*}$ & 100 & 109 \\
\hline Mas de 2300 & $33^{*}$ & 181 & 214 \\
\hline total & 42 & 281 & $323^{* *}$ \\
\hline
\end{tabular}

* $p<0,05 \quad$ ** un caso sin el dato. 
aportados en las publicaciones científicas respecto a la in-cidencia de los hematomas postlitotricia es si el estudio realizado tiene base experimental con modelos animales, en cuyo caso se ha reportado cifras del 40\% (21,22); también el tipo de litotritor parece influir, (20), p. ej., se han observado diferencias significativas al comparar el EDAP LT-01 con el Lithostart Siemens $(16,6 \%$ vs $19,6 \%)$. Por todo lo anterior creemos que la incidencia constatada en el presente estudio $(13 \%)$ es compatible con la experiencia científica existente.

La incidencia acumulada de los HP resultante en el presente estudio, $(2,16 \%)$, que difiere significativamente de la prevalencia encontrada a las $24 \mathrm{hrs}$. $(1,23 \%)$, debe ser destacada por su importancia clínica asistencial, e incluso potencialmente legal, debido a la necesidad de seguimiento que implicaría en los días posteriores a la litotricia para determinado grupo de pacientes.

- Antecedentes de LEOCH ipsilateral: Aunque otros han relacionado este factor, (12), nosotros no hemos reproducido dicho hallazgo ni globalmente ni al subdividir a los pacientes según el intervalo de tiempo transcurrido desde la última litotricia.

- Localización del/os cálculo/s: Nuestros resultados respecto al predominio significativo de cálculos caliciales para los HS y HP, así como el carácter asintomático de la casi totalidad de los hematomas acontecidos en las litiasis piélicas podrían ser explicados por la acción sectorial de la onda de choque sobre el parénquima renal, mas acentuada a nivel calicial, así como la relativa avascularidad (foco mas alejado del parénquima) y el medio líquido que ofrece la pelvis renal lo que probablemente disminuye la susceptibilidad a esta complicación y posiblemente minimiza su magnitud potencial en caso de producirse.

- IMC: se ha señalado a la obesidad como factor predisponente debido a las dificultades para la colimación del foco al calculo (33), nosotros no encontramos relación entre el incremento del IMC y la aparición de hematomas (según el test de regresión logística), en concordancia otros autores que no han comprobado relación con el peso corporal $(16,34)$ aún así, cuando dividimos nuestros pacientes en dos categorías (IMC > 26,5 y IMC < 26,5) si apreciamos diferencia significativa para los HP a favor de los pacientes con sobrepeso, por lo que consideramos a este factor algo controvertido.

- Afecciones renales relacionadas con los hematomas: la alteración parenquimatosa local en los pacientes con hidrocaliz podría teóricamente justificar la susceptibilidad de lesión microvascular, sin em- bargo, la asociación de las litiasis de cistina, (cistinuria comprobada), con los hematomas nos indujo a un análisis mas detallado y comprobamos que en todos los casos la energía acumulada superó la cifra de $150 \mathrm{~J}$, siendo en el $75 \%$ de los mismos mayor de 160, (media = 174,25), así mismo, también en el $75 \%$ de los portadores de litiasis cistina el número de impactos fue mayor de 2300 (media = $2452,8)$; recordemos que estas cifras se relacionaron estadísticamente con la aparición de hematomas en el análisis general de nuestra casuística; además se observaron en ellos las siguientes circunstancias concomitantes: HTA perioperatoria en el 50\%, TTPA ligeramente elevada (44 seg.), LEC ipsilateral 10 días antes y antecedentes de HTA en el $25 \%$, finalmente es llamativo el hecho de que en el paciente donde el número de ondas fue menor, se aplicó la mayor intensidad, (18,5 KV); todo ello nos lleva a la conjetura de que en estos pacientes, portadores de litiasis de mayor dureza, la necesidad de mayor numero de ondas $y / 0$ intensidad, en presencia de cofactores influyentes, aumentaría las probabilidades de presentar un hematoma renal postlitotricia, todo lo cual avala la hipótesis de que la aparición de este no solo es multifactorial sino que depende de la concurrencia de varios factores en un mismo paciente. Se ha reportado en la literatura médica una incidencia del $20 \%$ de hematomas en pacientes con litiasis de cistina los cuales necesitaron de una a cuatro sesiones (27), en nuestros pacientes dicha incidencia ha sido del $50 \%$.

- Síntomas y signos: Los aspectos relacionados con el cuadro clínico del hematoma renal postlitotricia se exponen en el apartado de resultados, es evidente que el síntoma dominante es el dolor y que existen carácterísticas que pueden orientarnos en el diagnóstico del tipo de hematoma, pero debemos destacar el caso de la paciente cuadriplégica comentado en el mismo, en el cual, la ausencia de dolor y de cambios en sus constantes vitales (paciente habitualmente hipotensa), así como la imagen de dudoso pequeño hematoma propiciaron el alta, pero su evolución ulterior nos confirma que en pacientes con características especiales como esta, con alteración de las vías sensitivas y posibles alteraciones vasomotoras por su patología de base, las primeras $72 \mathrm{hrs}$. son importantes para la observación de un posible HP. Otro aspecto a comentar es la relación estadística evidenciada de la dilatación pielocalicial con los HS, dicha ectasia podría haber influido en el momento de clasificar a estos pacientes como hematomas sintomáticos ya que el dolor pudo deberse a la obstrucción o dicha circunstancia haber potencializado dicho síntoma, luego, existe la posibilidad de que este grupo esté sobrevalorado, no obstante cabe aclarar que hemos tenido en cuenta siempre el descartar los dolores tí- 
picos de cólico nefrítico (ritmo, irradiación y cortejo vagal) asociados a dilatación pielocalicial sin imágenes ecográficas sugestivas de hematoma y con restos litiásicos que la justificara.

- Tamaño de los hematomas: En relación con el tamaño de los hematomas nuestros hallazgos están en sicronía con los de otros autores al constatarse que el $19 \%$ de los mismos tuvieron diámetros que abarcaban un tercio o mas del tamaño renal, $(23,24)$. En nuestros pacientes la asociación estadística del mayor tamaño con el grupo de HS estuvo determinada casi totalmente por la presencia de dolor, lo cual consideramos que está en correspondencia con la mayor distensión capsular en estos pacientes.

La valoración de los datos resultantes en el análisis estadístico parecen sugerir $(p<0,06)$ que el tamaño inicial podría tener interés como predictor de la progresión del hematoma ya que el $85,7 \%$ de los HP tuvo ambos diámetros iniciales de 4 o más cms.

- Imagen ecográfica: En 26 casos la imagen ecográfica (asociada o no a síntomas) ofreció dudas sobre la presencia de extravasación difusa intraparenquimatosa, con lo que podemos decir que, (teniendo en cuenta los 40 hematomas observados en la ecografía inicial y que 2 con imagen no concluyente fueron finalmente diagnosticados como tal), en 66 unidades renales tratadas, $(20,3 \%)$, se observó algún tipo de alteración ecográfica que según las urografías y/o ecografías aportadas por los pacientes y sus antecedentes patológicos, presumiblemente no se debieron a patología renal previa y por ende probablemente relacionada con la acción de las ondas de choque sobre el riñón; es por ello que tuvimos en cuenta la incidencia de los HP en estos casos (Gráfico 2 y $6 \mathrm{~A}$ ). En este sentido es conocida la alta frecuencia con que diversos autores han comprobado alteraciones renales tales como la pérdida de la diferenciación corticomedular entre el 20 y el $40 \%$ de los casos con métodos mas sensibles como la RMN, cifra que puede elevarse al 50 - $63 \%$ si se incluyen los hematomas $(10,17,21,25,26)$; otros han reportado cambios imagenológicos hasta en el $74 \%$ de los pacientes y explican dichos cambios por el edema y extravasación de orina $y / o$ sangre en el intersticio y espacios subcapsulares o perirrenales $(17,26)$; de acuerdo con ello creemos que nuestros resultados en el aspecto imagenológico son superponibles a los en la literatura teniendo en cuenta las limitaciones propias de la ecografía, por ende consideramos que debido a su accesibilidad es una herramienta indiscutible para la evaluación renal postlitotricia en casos clinicamente sospechosos o con factores de riesgo asociados; no obstante, el hecho de que en el $28,6 \%$ de los casos cuyo hematoma evolucionó de forma progresiva, la ecografía inicial no ofreció imágenes convincentes de hematoma, nos demuestra que, aunque sea un medio útil y asequible, la valoración de estos pacientes debe ser integral, sin subestimarse en ningún momento los aspectos clínicos (síntomas, signos y factores de riesgo presentes) para insistir en el seguimiento ecográfico, o apelar oportunamente a estudios mas sensibles y específicos como la TAC o la $\mathrm{RMN}$ en casos dudosos.

- Antiagregantes plaquetarios, enoxoparina y AINES: El uso de anticoagulantes $y$ antiagregantes ha sido reiterado en la literatura como factor de riesgo potencial $(19,28-31)$ es por ello que en nuestra unidad exigimos la suspensión de los antiagregantes plaquetarios antes de la litotricia, (preferiblemente siete días para la aspirina y cinco para el resto); sin embargo, en algunos casos fué necesaria la sustitución por heparina fraccionada y esta generalmente no se administró el día de la litotricia, a pesar de ello comprobamos la asociación de estos fármacos con la evolución progresiva de los hematomas, lo cual sugiere nuevas interrogantes, ¿̇son suficientes estas medidas?, ¿̇serán significativo el tipo o principio activo del fármaco, las dosis y el tiempo previo de tratamiento?, ¿influye la gravedad de la microvasculopatía asociada en los casos correspondientes? ...; en todo caso estas serían incógnitas a despejar en nuevos estudios controlados con mayor número de casos.

En el presente estudio analizamos, en los 28 pacientes que llevaban tratamiento habitual con antiagregantes $y / o$ anticoagulantes, el tipo de antiagregante, la velocidad de progresión del hematoma en estos pacientes vs pacientes sin dicho tratamiento previo, el uso de enoxoparina, la TA perioperatoria, y enfermedades asociadas; como resultado de dicho análisis hemos comprobado que en aquellos pacientes que llevaban tratamiento con antiagregantes 0 anticoagulante la evolución progresiva de los hematomas se produjo mas precozmente (en las primeras $18 \mathrm{hrs}$.), mientras que en los que no cumplían esta característica la progresión clínicamente significativa fué mas tardía (a partir de las 48 hrs.), esta curiosa situación puede ser el resultado de la mayor predisposición de estos enfermos al sangramiento, lo que pudo condicionar la cuantía inicial o la mas rápida evolución del mismo. No resultó significativo el tipo de antiagregante previamente utilizado ni el tipo de enfermedad asociada, pero si el hecho de que tres de los cuatro pacientes que tenían pautado enoxoparina tras la suspensión o "solapamiento" de los antiagregantes, presentaron hematomas $(75 \%)$, de los cuales, dos, presentaron HP; en correspondencia, la asociación de enoxoparina preoperatoria con los HS y HP resultó estadísticamente significativa $(p<0,05$ 
y $\mathrm{p}<0,02$ respectivamente, ver Tabla $\mathrm{VI}$ ), para el resto de los subgrupos, en el caso de los HS existió la mayor frecuencia de consumo de antiagregantes pero en el análisis estadístico no alcanzó suficiente relación $(p<0,09)$.

No hubo diferencias dentro de este grupo de pacientes ("consumidores habituales de antiagregantes") con respecto al comportamiento de la TA perioperatoria como cofactor en virtud de la aparición de hematomas ni de la progresión de estos pero si la hubo cuando se vincula la HTA pre o postlitotricia al uso de enoxoparina por lo que al parecer la suspensión de los antiagregantes antes de la litotricia podría ser suficiente en estos casos pero no podemos descartar que en el uso de anticoagulantes, (enoxoparina), en sustitución de los primeros sea un factor predisponente adicional teniendo en cuenta que su actividad en plasma puede prolongarse hasta 24 hrs., o, desde otro punto de vista, por ser el resultado de un régimen antiagregante previo mas intenso (dosis) y riguroso, o de un estado vascular mas precario en los pacientes a los que se prescribe esta pauta eludiéndose por imperativo la suspensión temporal absoluta de medicación antiagregante 0 anticoagulante, días antes de la litotricia.

Se han reportado hematomas retroperitoneales en pacientes tratados con enoxoparina a pesar de ser considerada un fármaco seguro, (32). Debido a que la biodegradación de la enoxoparina se efectúa principalmente en el hígado, la posibilidad de trombocitopenia y hemorragias existentes como reacciones adversas, y teniendo en cuenta también la posible asociación de las hepatopatías o alteración de las transaminasas, evidenciadas en presente estudio, y señaladas ya por otros autores, con los hematomas postlitotricia $(28,33)$, debemos insistir en la necesidad de mayores precauciones en los pacientes sometidos a litotricia en los que se detecten o se sospechen dichas situaciones. Respecto a la enoxoparina otros autores han recomendado precaución con la HTA no controlada y ante enfermedades renales ya que, incluso en disminuciones leves del aclaramiento plasmático de creatinina, se produce acumulación de esta en sangre potencializándose el efecto anticoagulante (32).

Al incluir a pacientes consumidores importantes y habituales de ibuprofeno y piroxican en el grupo de los pacientes tratados con antiagregantes la relación con el grupo HS se hizo significativa $(p<$ 0,05 ); a la luz de este resultado no debemos pasar por alto ciertas características de los AINES y la frecuencia con la que son utilizados en general e incluso en el síndrome doloroso del paciente litiásico y como parte de los protocolos analgésicos postlitotricia, es por ello que creemos necesarias algunas observaciones:

Los AINES, al inhibir a la cicloxigenasa promueven la formación de tromboxanos $\mathrm{A} 2$ en las plaquetas y de prostaciclina en el endotelio, ambos con acción antiagregante $o$ antiadhesiva plaquetaria $(34,35)$, también disminuyen la síntesis de prostaglandinas (PGs.), el filtrado glomerular y aumentan la liberación de renina, todo lo cual se potencializa con el uso prolongado de estos y propicia susceptibilidad a presentar HTA, incluso disminuyendo la acción de algunos antihipertensivos, (36-38). Ya hemos observado la importancia de la HTA perioperatoria en la aparición de hematomas y de su evolución progresiva, no menos importante es el hecho de que estos medicamentos se metabolizan en el hígado y se excretan por vía renal (35-37), no podemos olvidar que estamos tratando a pacientes que con frecuencia presentan una uropatía obstructiva y que se ha evidenciado en nuestros resultados que las hepatopatías y el incremento de las transaminasas son factores relacionados con la aparición de hematomas postlitotricia y que existen datos que avalan la posible hepatotoxicidad inducida por AINES como el diclofenaco y el acecoflenaco, (39), por último, y como colofón, debemos tener en cuenta que los AINES, incluyendo el ketorolaco (muy frecuentemente utilizado por nosotros) pueden interactuar con la enoxoparina, cuya asociación con los hematomas en el presente estudio ya fue comentada anteriormente, aumentando por tanto el riesgo de hematomas $(32,40)$.

A excepción de la aspirina, el ibuprofeno y el piroxican tienen un efecto antiagregante reversible pero difieren en el tiempo de recuperación de la actividad plaquetaria, variando este entre $24 \mathrm{hrs}$. y dos semanas respectivamente, (40) aspecto este que debe ser tenido en cuenta (conjuntamente con los datos del perfil funcional hepático y renal) para valorar el riesgo de hematoma, sobre todo si el paciente necesita tratamiento analgésico postlitotricia, (p.ej. ketorolaco, diclofenaco, etc.), o presenta HTA.

Los AINES también interactúan con la furosemida potencializando el incremento de la actividad plasmática de la renina que esta induce, (40), por lo que sería un factor añadido a tener en cuenta en las unidades de litotricia en las que, como en la nuestra, este diurético se utiliza frecuentemente en la fase final de la sesión de litotricia.

Atendiendo a los comentarios anteriores una vez mas se evidencia que en el manejo de estos pacientes debemos tener presente diferentes factores por su latente interrelación propiciadora de hematomas. 
- Diagnóstico previo de HTA y comportamiento perioperatorio de la TA: varios autores han relacionado a la HTA con los hematomas postlitotricia (ver Tabla III), sin embargo nosotros no encontramos relación de estos con el diagnóstico previo de dicha entidad como factor independiente, por ejemplo, de los 42 hematomas diagnósticados solo 6 pacientes $(14,2 \%$ de los hematomas), tenían diagnóstico previo de HTA sin otra enfermedad asociada, de los cuales 4 presentaron cifras preoperatorias elevadas y 1 HTA pre y postoperatoria, así mismo de los 80 casos con diagnóstico previo de HTA, (con o sin otras enfermedades asociadas), solo 8 $(10 \%)$ presentaron hematomas; otro dato ilustrativo es el hecho de que en el grupo de los HP solo un paciente era hipertenso conocido, siendo este el único que presentó HTA postlitotricia; por tanto, de acuerdo con los correspondientes resultados parece mucho mas importante el comportamiento de la TA pre y postoperatoriamente; en este sentido algs. autores han llamado la atención sobre el mal control de pacientes hipertensos como factor agravante, $(15,16)$, y otros destacan el incremento de la TA en el preoperatorio por encima de 160/95 (43) aspectos estos que el presente trabajo corrobora; es posible que la hipertensión perioperatoria haya sido constatada en pacientes hipertensos "no conocidos", "hiperreactivos" o incluso sujetos a interacciones medicamentosas como las expuestas anteriormente en relación con los AINES.

En 23 casos, (7\%), se observó hipotensión (TA $\leq 100 / 60$ ) en las primeras $6 \mathrm{hrs}$. del postoperatorio, de los cuales 8 , o sea, un tercio, tenían hematoma, pero solo uno de estos tuvo signos de progresión; esta circunstancia nos llevó a la observación de que dicha disminución de la TA es en la mayoría de los casos transitoria, no significativa y probablemente secundaria a la sedoanalgesia, reflejo vagal o a la utilización de diuréticos transoperatoriamente, por lo que no constituye por si sola un dato indicativo de hematoma, lo cual nos obliga a enfatizar una vez mas el valor de la evaluación clínica integral, así como la identificación previa de los casos de riesgo para el diagnóstico oportuno de estos pacientes.

\section{Asociaciones de enfermedades concurrentes no re-} nales relacionadas con los hematomas: otro aspecto interesante es el significado estadístico encontrado en la relación de la asosiación de la cardiopatía isquémica con HTA o hepatopatías con los $\mathrm{H}$ y también en el grupo de los HS (no en los HA), hallazgos que apoyan una posible "hipótesis multifactorial sumatoria"; la enfermedad coronaria ya ha sido señalada como factor de riesgo por otros, (41), también la hipercolesterolemia y la arteriosclerosis avanzada (13, 30) y es conocida la relación causa efecto de estas entidades.
Microhematuria preoperatoria: en el 64,3\% (27 pacientes) de los hematomas se comprobó presencia de microhematuria preoperatoria resultando estadísticamente significativa dicha asociación $(P=0,04)$, no encontramos explicación convincente ante este hallazgo debido a su justificación por la teórica acción erosiva del calculo sobre el urotelio, aunque dicha microhematuria, a juzgar por los resultados, sí podría ser indicativa de una mayor propensión de estos enfermos al sangrado, en cuanto a la etiología inflamatoria o infecciosa de la misma no está respalda por no haberse encontrado relación estadística entre los urocultivos positivos, la leucocituria y los hematomas en el presente estudio.

Relación de transaminasas con hematomas: dentro de nuestros resultados es llamativa la correlación directa entre los valores ascendentes de TGO y GGT y la aparición de hematomas, si tenemos en cuenta la relación evidenciada entre las hepatopatías crónicas y los hematomas sintomáticos ya comentada con anterioridad ello nos acerca a una probable relación con las enfermedades hepatoesplénica subyacentes como factores predisponentes con efectos sobre la coagulación $(28,33)$, pero mas que afecciones hepáticas concretas asociadas, una de las hipótesis posibles sería la concurrencia de disfunciones o estados hepatoestructurales secundarios a noxas tales como la ingestión habitual de bebidas alcohólicas, fármacos hepatóxicos e hiperlipemia, entre otros; y aunque la comprobación de este supuesto escapa a los objetivos del presente estudio no deja de ser sugerente la asociación de elevaciones en las cifras de transaminasas con los hematomas de la presente serie independientemente de las hepatopatías encontradas.

Comportamiento de $\mathrm{Hb}$ y Hto.: el descenso de la $\mathrm{Hb}$ y el Hto. no se asoció a la inestabilidad hemodinámica por lo que consideramos que esta última no debe ser la clave para adoptar la decisión de indicar una transfusión oportuna ya que al parecer se trata de un cuadro "tardío"; por tanto, el seguimiento estricto de dichos parámetros hemáticos con carácter obligatorio aún cuando el paciente se encuentre hemodinámicamente estable, es muy importante. servados;

Creemos necesario destacar dos hechos ob-

Primero: aún cuando se alcancen valores aceptables a las $12 \circ 18 \mathrm{hrs}$ del diagnóstico cabe esperar descensos a las $24 \mathrm{hrs}$. aunque el paciente haya sido transfundido, y no es posible asegurar una estabilización de estos parámetros hasta que transcurran al menos 48 hrs. desde el diagnóstico y tratamiento del hematoma sintomático de curso progresivo; 
Segundo: la respuesta postransfusional resultó ser escasa, (Tabla VII), lo cual probablemente se beba a la redistribución volumétrica hemática tras una pérdida inicial importante o a un descenso gradual del sangramiento en las horas posteriores, en nuestros pacientes no pretendimos lograr valores de $\mathrm{Hb}$ y $\mathrm{Hto}$. óptimos sino niveles estables, no obstante no podemos soslayar el hecho de que en tres pacientes la progresión, aunque mas lenta, existió dentro de los cinco días posteriores al alta hospitalaria lo cual nos obliga a resaltar la importancia del seguimiento de los pacientes con HS o de alto riesgo durante la primera semana del postoperatorio.

Influencia de los parámetros técnicos de la litotricia: probablemente el apartado tecnico-terapéutico haya sido el mas estudiado para los hematomas postlitotricia y a su vez el mas controvertido, unos autores destacan como factor predisponente el número de ondas, $(8,10,19,22)$, el tipo de litotritor o generador de ondas, $(20,44,46)$, otros destacan las ventajas de la onda doble sincrónica sobre la estandar (45), mientras que la intensidad también ha sido relacionada (39); sin embargo existen estudios en los que no se ha encontrado relación significativa entre estos parámetros y la aparición de los hematomas o el mayor daño renal tisular $(15,18,43,45,46)$.

Uno de los aspectos menos abordados es la energía total, se ha valorado la influencia de la misma y no se halló relación (43), nosotros al estratificar los datos observamos asociación cuando la misma supera los $150 \mathrm{~J}$ (mas de $160 \mathrm{~J}$ para los HP). En el caso del número de ondas, comprobamos que para cifras mayores de 2300 impactos se evidenció asociación con los hematomas en general $(p<0,05)$ y en particular para el grupo de los HS, por otra parte todos los HP recibieron mas de 2.300 impactos de ondas, sin embargo, cuando intentamos relacionar este parámetro con variables que demostraron asociación con la aparición de hematomas (HTA pre y postoperatoria, o uso de anticoagulantes), ninguna de ellas evidenció estadísticamente que al asociarse con mas de 2300 ondas aplicadas la incidencia de hematomas en general, HS o HP fuera mayor, lo cual refleja independencia entre este parámetro y las variables mencionadas; por todo ello creemos que las discrepancias entre los resultados de los diferentes autores se deben posiblemente a la combinación entre los parámetros técnicos utilizados, a otras posibles covariables influyentes y al proceso estadístico seguido, así mismo no es descartable el valor potencial de dichos parámetros tecnicoterapéuticos en función del equipo disponible en cada unidad de Litotricia. En cuanto a la intensidad creemos que el rango en el que nuestros pacientes fueron tratados no propició la conformación de grupos estrictamente comparables, ya que el $88 \%$ de los casos se les aplicó intensidades entre 17,5 y $18,5 \mathrm{KV}$, no obstante es destacable el hecho de que en los 19 unidades renales tratadas con intensidad inferior a $17 \mathrm{KV}$ solo se observó un hematoma renal $(5,26 \%)$ en contraste con los 41 hematomas diagnosticados en las $305(13,44 \%)$ en las que se superó dicha cifra; al respecto se ha señalado que intensidades entre 14 y $16 \mathrm{KV}$ con el Modulih SL 20 de generador electromagnético se relacionaron con la aparición de hematomas subcapsulares y los valores entre 17 y $20 \mathrm{KV}$ con hematoma perirrenal (47), el autor observó además que el número de ondas no fue importante a intensidades bajas o medias (11 a $13 \mathrm{KV}$ ), pero si lo era a intensidades mayores, sobre todo cuando esta era de $20 \mathrm{KV}$ en cuyo caso el mayor número de impactos se correlacionó con los hematomas perirrenales mientras los corticales aparecían con menor números de ondas; nuestros resultados nos permiten recomendar en pacientes con varios factores de riesgo valores de intensidad de $16,9 \mathrm{KV}$, (correspondientes, en el generador electromagnético tipo $220 \mathrm{fXXP}$ del litotritor DOLI-S, al nivel de intensidad 9), ya que con el mismo no se observó incidencia de hematomas progresivos (todos aparecieron con intensidades entre 17,5 y $18 \mathrm{KV}$ ), solo apareció un hematoma sintomático y la incidencia total de hematomas cae en mas del $50 \%$.

Por último es necesario aclarar que en nuestra unidad la frecuencia de impactos utilizada es de 70 u 80 / minuto, incluso en una misma sesión en mas de la mitad de los casos, sobre todo después que se presentó el caso que motivó el presente estudio, es por ello que en nuestra serie solo se utilizó frecuencia de 60 en tres niños, (ninguno presentó hematoma); por lo que este parámetro, que consideramos importante, no observó ningún comportamiento estadístico digno de destacar.

En resumen y consecuentemente con estas observaciones, a luz de nuestros resultados, podemos sugerir que: una correcta correlación entre el número de ondas y la intensidad seleccionada, teniendo en cuenta las características clínicas del paciente y los posibles factores de riesgo asociados sería un criterio mas razonable que plantearse disminuir la incidencia de los hematomas clínicamente significativos en función de estrategias dirigidas a parámetros o factores aislados.

Pacientes con mayor riesgo de hematomas significativos: En todos los casos en los que se produjo HP concurrieron tres o mas de los factores que en el presente estudio se encontraron asociados estadísticamente, un caso ilustrativo es el de la paciente que requirió reingreso a los 5 dias de la litotricia en la que concurrieron siete condicionantes, estos fueron: 
HTA pre y postoperatoria, aumento de transaminasas, microhematuria, número de impactos superior a 2300 , energía acumulada mayor de 160, medicación de efecto antiagregante plaquetaria, así como circunstancias que pudieran tener cierta relevancia como diámetros iniciales del hematoma mayor de 4 $\mathrm{cms}$ (posible indicativo de mayor probabilidad de progresión), IMC mayor de 26,5 (controvertido), el antecedente de anemia ferropénica crónica y leucocituria (como agravantes de la repercusión sistémica del hematoma). Probablemente en los pacientes portadores de los hematomas postlitotricia de mayor importancia clínica, existe un "efecto sumatorio" de los factores evidenciados en el presente trabajo e incluso de otros señalados por diferentes autores. En consecuencia con nuestros resultados deben ser considerados también: litiasis caliciales, mas de una litiasis, fármacos habituales (antiagregantes plaquetarios, AINES, enoxaparina, e incluso, en algún paciente, los potenciales hepatotóxicos como la hidantoía y el fenobarbital observados en dos de nuestros pacientes, uno de los cuales presentó HP), hepatopatía crónica aislada o asociada con cardiopatía isquémica, HTA asociada con cardiopatía isquémica, litiasis en hidrocaliz (posible mayor predisposición local), litiasis cistínica (necesidad de mayor número de disparos e intensidad), e intensidad superior a $17 \mathrm{KV}$.

\section{Evolución sintomática a mediano y largo plazo (de 7}

a 19 meses): Como se recoge en nuestros resultados hay que tener en cuenta que un grupo de pacientes se mantienen dolor lumbar incluso meses posteriores a la litotricia, probablemente dicho dolor está en relación con el proceso fibrótico irritativo perirrenal sobre terminaciones nerviosas capsulares e incluso sobre los nervios iliohipogástrico e ilioinguinal en aquellos pacientes con irradiación anteroinferior del mismo.

Manejo terapéutico de los hematomas: aunque el caso que motivó el presente estudio requirió una embolización arterial selectiva, el tratamiento de todos los casos incluídos en esta serie fue conservador, lo que está en concordancia con la experiencia universal dentro de la cual es muy infrecuente la necesidad de tratamiento invasivo (embolización o cirugía). La mayoría de nuestros pacientes respondieron bien a medidas clásicas tales como sueroterapia (con o sin expansores plasmáticos, analgesia escalonada, transfusión de hematíes concentrados (en casos con descenso significativo de $\mathrm{Hb}$ y Hto., representando solo el $7,1 \%$ del total de hematomas) y reposo físico; sin embargo, en tres casos fueron necesarias medidas adicionales como el uso de furosemida, ansiolítico y Acido Epsilon Aminocaproico e hipotensores (este último solo en un paciente); la furosemida la indicamos en pacientes cuyo incremento de la tensión arterial postlitotricia es mantenido y se convierte en un factor potencial de perpetuación del sangramiento, posee el doble mecanismo teórico de mejorar la perfusión renal, aumentando la diuresis y disminuyendo el edema y la distensión capsular, su efecto hipotensor puede verse disminuído si se utilizan AINES, (40), lo cual debe tenerse en cuenta si concomita hipertensión postoperatoria y no se controla con esta medida, por otra parte, en el paciente politransfundido la furosemida disminuye la posible hiperpotasemia. El diazepan y la prometacina contribuyen a disminuir la ansiedad generada por el dolor, factor que puede agravar la HTA o provocar inestabilidad hemodinámica, el segundo además atenúa los efectos eméticos de la meperidina rutinariamente utilizada por nosotros ante el dolor incontrolable; el epsilon aminocaproico influye, al menos en teoría, en la detención de la hemorragia, en el caso del ansiolítico y del diurético es necesario tener en cuenta el estado hemodinámico y hemático (Hb y Hto.) a fin de evitar potenciar los efectos de la hipovolemia y la hipoxia; en nuetros pacientes estas medidas resultaron eficaces aunque es prudente señalar que uno de estos reingresó al tercer día del alta por progresión del hematoma y dolor después de haber sido dado de alta a las $48 \mathrm{hrs}$. de la litotricia tras 24 hrs. de estabilidad hemática, ecográfica y sin dolor, aunque no fue necesaria una nueva transfusión.

\section{CONCLUSIONES}

- La incidencia de los hematomas renales postlitotricia en el presente estudio fue del $13 \%$, pero solo se constató hematomas sintomáticos en el $6,2 \%$ de las litotricias realizadas y la prevalencia de los de curso progresivo fue del $2,16 \%$.

- En el $20,4 \%$ de las unidades renales tratadas con el litotritor DOLI-S se observaron alteraciones ecográficas en el parénquima renal probablemente secundarias a las ondas de choque.

- La ecografía renal para el diagnostico inicial de los hematomas resultó dudosa en el $28,57 \%$ de los hematomas de evolución progresiva, o sea, especificidad del $71,4 \%$, por lo que dicho estudio, aunque muy útil y asequible, no es completamente seguro como único método imagenológico para diagnosticar o determinar la incidencia real de esta complicación, imponiéndose en ocasiones la necesidad de apelar a otros con mayor especificidad en determinados casos sintomáticos (TAC en dos de nuestros pacientes), para un diagnóstico mas exacto.

- Un 6,8\% de los casos tratados pudieron ser dados de alta sin sospecharse existencia de hematoma renal o perirrenal por ser asintomáticos en la primeras 22 o 24 hrs. 
- Algunos pacientes dados de alta a las 24 horas sin sospecha de hematoma evolucionan de forma progresiva en los días ulteriores requiriendo reevaluación médica; en nuestra serie esta situación se presentó en el 0,6\% de las litotricias realizadas.

- En el $25 \%$ de los hematomas progresivos diagnósticados en las primeras $12 \mathrm{hrs}$. la estabilización clínica, hematológica y ecográfica durante 24 hrs. no garantizó la estabilidad evolutiva y por ende sintomática en los días posteriores al alta.

- El dolor fué el principal síntoma del hematoma renal postlitotricia (100\% de los HS), y tuvo como rasgos distintivos los siguientes: intenso, constante (subcapsulares) o irregular (perirrenales), lumbar (subcapsulares) difuso (perirrenales) y de localización ocasionalmente atípica como hombro, precordio y dorsal infraescapular. En pacientes cuadriplégicos el dolor no es un indicativo clínico fiable.

- Los hematomas renales y perirrenales pueden ser distinguibles semiológicamente, aunque algunos hematomas inicialmente pueden ser subcapsulares y evolucionan con componente perirrenal a posterioris en cuyo caso dicha distinción puede perder valor.

- La ubicación calicial de la litisis tratada, (principalmente caliz inferior), se relaciona con hematomas sintomáticos y la piélica con hematomas asintomáticos.

- La hipotensión arterial no fue un signo indicativo de hematoma, ni de su volumen, en las primeras horas postlitotricia, por lo que la inestabilidad hemodinámica debe considerarse como un cuadro tardío o asociado a hematomas de gravedad extrema.

- La hipertensión arterial perioperatoria fue mas importante que el diagnóstico previo de HTA como factor predisponente para la incidencia de hematomas postlitotricia, este último solo tuvo valor cuando se asoció a cardiopatía isquémica o hepatopatía.

- Los hematomas que ecográficamente, al ser diagnosticados, presentan diámetros de $4 \mathrm{~cm}$ (aproximadamente $1 / 3$ del eje longitudinal) o mas, son significativamente mas propensos a evolucionar de manera progresiva.

- Los factores posiblemente predisponentes para la aparición de hematomas sintomáticos y progresivos después de la litotricia evidenciados estadísticamente en este estudio son: hepatopatías crónicas, elevación de las transaminasas, hipertensión arterial perioperatoria, asociación de HTA y cardiopatía isquémica, microhematuria preoperatoria, locali- zación calicial de la/s litiasis, el uso habitual de antiagregantes plaquetarios y/o AINES, sobre todo con pauta de sustitución perioperatoria por enoxoparina (para HP), número de ondas y energía total superiores a 2300 y $150 \mathrm{~J}$ (para HS) ○ $160 \mathrm{~J}$ (para $\mathrm{HP}$ ), el IMC mayor de 26,5 y la presencia de 2 o mas litiasis tratadas.

- Las intensidades mayores de $17 \mathrm{KV}$ parecen tener importancia pero este dato no fue suficientemente significativo $(p<0,07)$.

- La diabetes mellitus, la LEC ipsilateral, el sexo, el lado tratado, la uropatía obstructiva y su grado, no resultaron asociadas estadísticamente a la aparición de hematomas.

- Los pacientes portadores de litiasis de cistina y de hidrocaliz, según el presente estudio, parecen ser mas propensos a presentar hematomas postlitotricia. En el caso de los calculos de cistina probablemente por requerir mayor número de ondas e intensidad.

- En los "hematomas progresivos" la unidad renal predominantemente afectada fue la izquierda, esta y la localización del calculo en caliz inferior se observó en el $85,7 \%$ dentro de este subgrupo 16 de los 7 casos) y en el caso de la localización calicial, en el $55 \%$ de los "hematomas sintomáticos", siendo en ambos grupos significativamente estadística esta relación

- El uso de antiagregantes y enoxoparina, y la hipertensión arterial en las hrs. posteriores a la litotricia parecen condicionar, no solo la aparición de un HP, sino también la rápidez en la progresión del hematoma.

- En casos aislados (7\% de esta serie), el hematoma renal postlitotricia puede progresar durante los primeros 5 días a pesar de transcurrir de forma asintomática durante las $48 \mathrm{hrs}$. iniciales o haber permanecido estable clínica, hematológica y ecográficamente durante al menos $24 \mathrm{hrs}$. tras el diagnóstico y tratamiento inicial.

- De acuerdo con nuestros resultados el 1,54 \% de los casos tratados puede mantener dolor lumboabdominal durante meses después de haber presentado un hematoma renal postlitotricia, representando el $11,9 \%$ dentro de este grupo de pacientes.

\section{RECOMENDACIONES}

Derivadas del análisis de nuestros resultados y conclusiones podemos hacer las recomendaciones prácticas que resumimos en el siguiente Algoritmo: 


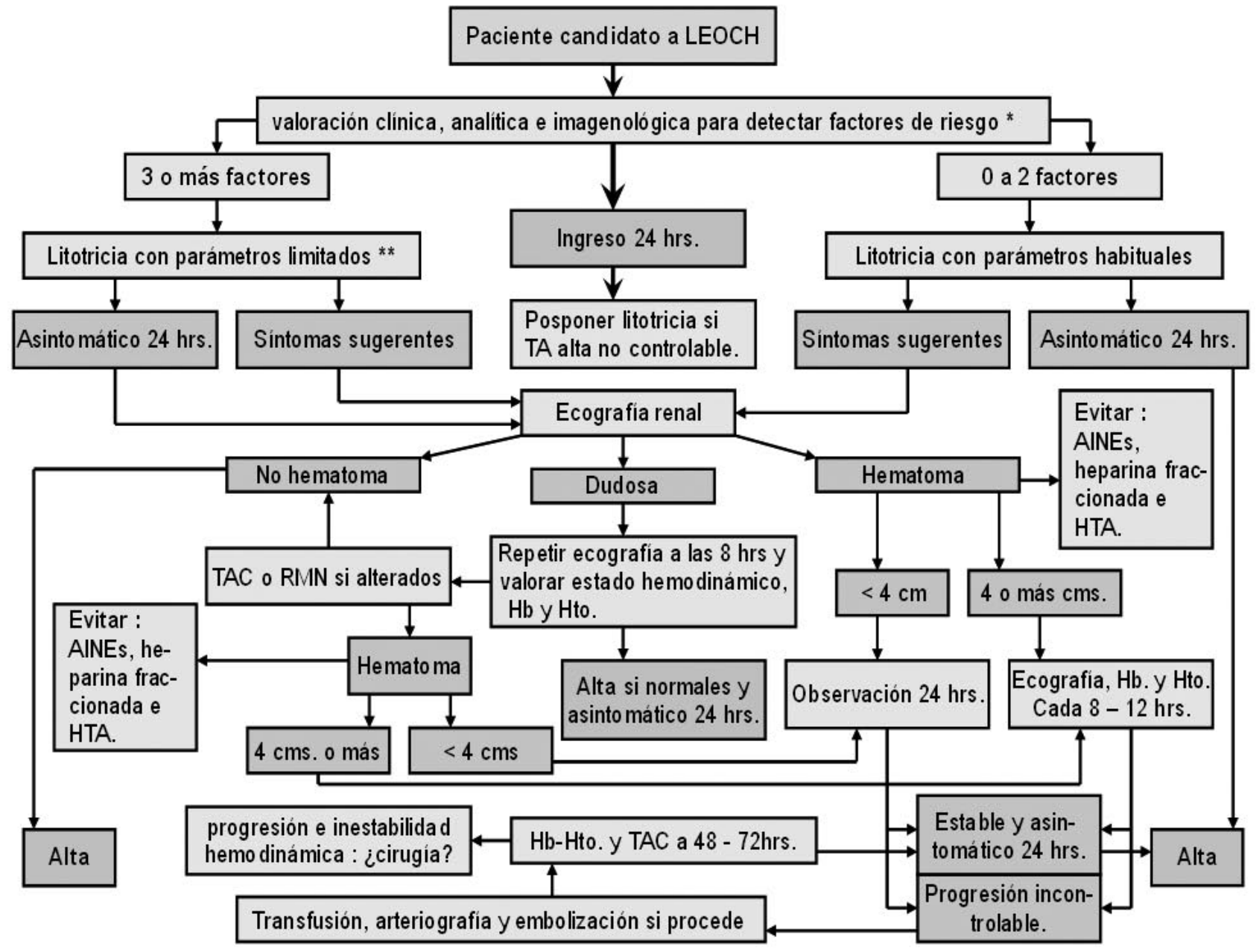

\section{*Factores o condiciones:}

-Uso habitual de antiagregantes tes, anticoagulantes o AINEs.

- Hepatopatía crónica.

- HTA + cardiopatía is quémica.

- HTA antes de la litotricia.

- Elevación de las transaminasas.

- Hematuria microscópica.

- Uso de heparina fraccionada-

24 hrs. antes.

- Litiasis calicial /es.

- Litiasis en hidrocaliz.

- Litiasis cistínica. ${ }^{\dagger}$

- Sobrepeso.†

\section{** Litotricia con parámetros limitados:}

Número de ondas: hasta 2300

Intensidad: hasta $17 \mathrm{KV}$

Energía total: hasta $160 \mathrm{~J}$

Frecuencia: 70 a $80 /$ min. 


\section{BIBLIOGRAFÍA y LECTURAS RECOMENDADAS ( ${ }^{*}$ lectura de interés $y^{* *}$ lectura fundamental)}

1. RUIZ MARCELLAN, F.J.; IBARZ SERVIO, L.: "Historia de la litotricia por ondas de choque en España”. Arch. Esp. Urol., 8: 1003, 2007.

2. LARREA MASVIDAL, E. y cols.: "Tratamiento de la litiasis renoureteral mediante litotricia extracorpórea por ondas de choque. Experiencia en Cuba”. Arch. Esp. Urol., 1: 109, 1989.

**3. TORRECILLA, O.C. y cols.: "Hematoma renal después de litotricia extracorpórea por ondas de choque". Actas Urol. Esp., 8: 752, 1997.

4. TORRECILLA, O.C. y cols.: "Tratamiento ambulatorio sin anestesia de la litiasis urinaria mediante litotricia por ondas de choque: 7000 casos". 1: 47, 1993.

5. BEATRICE, J. y cols.: "Life-threatening complication alter right renal extracorporeal shock wave lithotripsy: Large hepatic haematoma requiring embolisation of the right hepatic artery". Eur. Urol., 2: 16, 2007.

6. EGILMEZ, T. y cols.: "Efficacy and safety of a new-generation shockwave lithotripsy machine in the tretment of single renal or ureteral stones: Experience with 2670 pacients". J. Endourol., 1: 23, 2007.

*7. FISCHER, C. y cols.: "Extracorporeal shockwave lithotripsy induced ultrastuctural changes ot the renal perenchyma under aspirin use: Electron microscopic findings in the rat kidney". Urologe A., 1: 13, 2007.

*8. DELIUS, M. y cols.: "Biological effects of shock waves: Kidney damage by shock waves in dogsdose dependence". Ultrasound Med. Biol., 2: 117, 1988.

9. WEBER, C. y cols.: "Injury of rat vessels following extracorporeal shock wave treatment". J. Urol., 2: 476, 1992.

10. RECKER, F. y cols.: "Morphological changes following ESWL in the rat kidney". Urol. Res., 4: 229, 1989.

11. BEX, A.: GOEPEL, M.; MOLLHOFF, S.: "Extensive retroperitoneal hematoma following extracorporeal shock wave lithotripsy with second generation lithotripter". Urol. Int., 1: 111, 1992.

12. COLLADO, S.A. y cols.: "Renal hematoma as a complication of extracorporeal shock wave lithotripsy”. Scand J. Urol. Nephrol., 3: 171, 1999.

13. GALLEGO, S.J.A. y cols.: "Hematomas renales después de la litotricia extracorpórea con el litotritor Lithostar multilineal de Siemens". Actas Urol. Esp., 1: 19, 2000.

14. KUPELI, B. y cols.: "Extracorporeal shock wave lithotripsy for lower caliceal calculi". Eur. Urol., 3: 203, 1998.
*15. KOSTAKOPOULOS, A. y cols.: "Subcapsular hematoma due to ESWL: Risk factors. A study of 4247 patients". Urol. Int., 1: 21, 1995.

16. KNAPP, P.M. y cols.: "Extracorporeal shock wave lithotripsy-induced perirrenal hematomas". J. Urol., 4: 700, 1988.

17. BAUMGARTNER, B.R. y cols.: "Kidney changes after extracorporeal shock wave lithotripsy: Appearance on MR imaging". Radiology, 163: 531, 1987.

18. BLANGY, S. y cols.: "Complications of the treatment of renal calculi by extracorporeal lithotripsy. Prospective evaluation of 80 treated kidneys". J. Radiol. 68: 619, 1987.

19. FUCHS, G.J.; DAVID, R.D.; FUCHS, A.M.: "Complicaciones de la litotricia extracorpórea por ondas de choque”. Arch. Esp. Urol. 1: 83, 1989.

*20. MOMOSE, A. y cols.: "Effect of pressure distribution of shockwave on renal hemorrage alter extracorporeal shockwave lithotripsy. Comparison of EDAP LT-01 and Siemens Lithostar". J. Endourol., 13: 165, 1999.

21. RECKER, F. y cols.: "Magnetic resonance imaging of acute and long-term alterations following extracorporeal shock wave lithotripsy in rats". 45 : 28, 1990.

*22. NEUERBURG, J. y cols.: "Effects of lithotripsy on rat kidney: Evaluation with MR imaging, histology and electron microscopy". J. Comput Assist Tomogr., 13: 82, 1989.

23. RUBIN, J.I. y cols.: "Kidney changes after extracorporeal shock wave lithotripsy: CT evaluation". Radiology, 162: 21, 1987.

24. GROTE, R.; DOHRING, W.; AEIKENS, B.: "Computed tomographic and sonographic detection of renal and perirrenal changes following shockwave lithotripsy." Rofo., 144: 434, 1986.

*25. SAIKO, Y. y cols.: "The effect of extracorporeal shock wave lithotripsy on kidney and adjacent tissue detected by magnetic resonance imaging". Hinyokika Kiyo, 41: 577, 1995.

26. KAUDE, J.V. y cols.: "Renal morphology and function inmediately after extracorpo real shock wave lithotripsy". AJR Am J Roentgenl, 145: 305, 1985.

27. SLAVKOVIC A. y cols.: "Extracorporeal shock wave lithotripsy for cystine urolthiasis in children: Outcome and complications". Int. Urol. Nephrol., 34: 457, 2002-2003.

*28. RUIZ MARCELLAN, F.J. y cols.: "Litotricia extracorpórea por ondas de choque en pacientes con trastornos de coagulación". Arch. Esp. Urol., 45: $135,1992$.

29. RUIZ, H.; SALTZMAN, B.: “Aspirin-induced bilateral renal hemorrage alter extracorporeal shock wave lithotripsy therapy: Implications and conclusions". 143: 741, 1990. 
*30. DOMÍNGUEZ, M.J.F. y cols.: "Hematomas renales secundarios a litotricia por ondas de choque". Arch. Esp. Urol., 50: 767, 1997.

31. BAHCECI, M. y cols.: "Serius clopidrogel associated renal hematoma in a type 2 diabetic patient with primary hyperparathyroidism alter extracorporeal shock wave lithotripsy". Saudi Med. J., 26: 1007, 2005.

32. MONTERRUBIO, U.J.; CORDOBA, L.A.; CORCHO, S.G.: "Hematoma retroperitoneal y enoxoparina en un síndrome coronario agudo sin elevación del ST". Medicina Intensiva, 8: 427, 2002.

33. CHEN, C.S. y cols.: "Subcapsular hematoma of spleen-a complication following extracorporeal shock wave lithotripsy for ureteral calculus". Changgeng Yi Xue Za Zhi, 15: 215, 1992.

34. SCHRÖR, R.: "Antiplatelet drugs. A comparative review”. Drugs, 50: 7, 1995.

35. SAVI, P. y cols.: "Importance of hepatic metabolism in the agregating activity of the thienopyiridineclopidogrel”. Biochem Pharmacol, 44: 527, 1992.

36. HARDMAN, J.G.; LIMBIRD, L.E.; GOODMAN, G.A.: "Goodman \& Goodman, las bases farmacológicas de la terapéutica". Ed. Mc GrawHill Interamericana, $10^{\mathrm{a}}$ ed., Mex., DF Vol 1, pág. 707-726, 2003.

37. FLOREZ, J.: "Farmacología Humana". Ed. Masson, $4^{a}$ ed., Barcelona, España, pág. 385-399, 2003.

38. DE LEEUW, P.W.: "Nonsteroidal anti-inflammatory drugs and hipertensión. The rsks in perspective". Drugs, 51: 179, 1996.

39. ZARAGOZA, A.; ALFONSO, V.; ROIG, E.: "Hepatotoxicidad inducida por AINES: Aceclofenaco y diclofenaco”. Rev. Esp. Enf. Dig., 87: 472, 1995.

40. USPDI.: "Información de medicamentos para el profesional sanitario". Ministerio de Sanidad y consumo, $14^{\mathrm{a}}$ ed., pág. 120, 123, 143, 1067, 1643. 1995.
**41. NEWMAN, L.H.; SALTZMAN, B.: "Identifying risk factors in development of clinically significant post-shoc-wave lithotripsy subcapsular hematomas". Urology, 38: 35, 1991.

42. MEZENTSEV, V.A.: "Extracorporeal shock wave lithotripsy in the treatment of renal pelvicalyceal stones in morbidly obese patiens". Int. Braz. J. Urol., 31: 105, 2005.

43. UEDA, S. y cols.: "Perirrenal hematomas caused by SWL with EDAP LT-01". J. Endourol., 7: 11, 1993.

44. MORRIS, J.S. y cols.: "A comparison of renal damage induced by varying modes of shock wave generation". J. Urol., 145: 864, 1991.

45. SHEIR, K.Z. y cols.: "Evaluation of synchronous twin pulse technique for shock wave lithotripsy: In vivo tissue effects". Urology, 62: 964, 2003.

*46. DHAR, N.B. y cols.: "A multivariate analysis of risk factors associated with subcapsular hematoma formation following electromagnetic shock wave lithotripsy". J. Urol., 172: 2271, 2004.

47. RASWEILER, J. y cols.: "Experimental basis of shockwave-induced renal trauma in the model of the canine kidney". World J. Urol., 11: 43, 1993.

48. RODRÍGUEZ, V.L. y cols.: "Tratamiento de los cálculos renoureterales mediante ESWL y técnicas endourológicas". Arch. Esp. de Urol., 1: 38, 1989.

49. FERNANDEZ, F.A., y cols.: "Hematoma perirrenal después de ESWL en un pa- ciente con Tumor renal de estadio T1". Arch. Esp. Urol., 47: 156, 1994.

50. TRINCHIERI, A. y cols.: "Urinary excretion of n-acetyl-glucosaminidase after extra-corporeal shockwave lithotripsy: A marker of renal tubule injury”. Arch. Ital. Urol. Nefrol. Androl., 61: 407, 1989.

51. ANTONIOU, N.K.; KARANASTASIS, D.; STENOS, J.L.: "Severe hemorrhage after schockwave lithotripsy". J. Endourol., 9: 239, 1995. 